\title{
New Inertial Projection Methods for Solving Multivalued Variational Inequality Problems Beyond Monotonicity
}

\author{
Chinedu Izuchukwu ${ }^{1}$ Yekini Shehu ${ }^{2,3}$ iD
}

Accepted: 13 January 2021 / Published online: 3 March 2021

(C) The Author(s) 2021

\begin{abstract}
In this paper, we present two new inertial projection-type methods for solving multivalued variational inequality problems in finite-dimensional spaces. We establish the convergence of the sequence generated by these methods when the multivalued mapping associated with the problem is only required to be locally bounded without any monotonicity assumption. Furthermore, the inertial techniques that we employ in this paper are quite different from the ones used in most papers. Moreover, based on the weaker assumptions on the inertial factor in our methods, we derive several special cases of our methods. Finally, we present some experimental results to illustrate the profits that we gain by introducing the inertial extrapolation steps.
\end{abstract}

Keywords Inertial methods · Multivalued variational inequalities · Projection-type methods $\cdot$ Continuous mapping $\cdot$ Armijo-type linesearch

\section{Introduction}

Assume that $C$ is a nonempty closed and convex subset of $\mathbb{R}^{N}$ and $F: C \rightrightarrows \mathbb{R}^{N}$ a multivalued mapping with nonempty values. The Multivalued Variational Inequality

Yekini Shehu

yekini.shehu@unn.edu.ng

Chinedu Izuchukwu

izuchukwu_c@yahoo.com; izuchukwuc@ukzn.ac.za

1 School of Mathematics, Statistics and Computer Science, University of KwaZulu-Natal, Durban, South Africa

2 Department of Mathematics, Zhejiang Normal University, Jinhua 321004, People's Republic of China

3 Institute of Science and Technology (IST), Am Campus 1, 3400, Klosterneuburg, Austria 
Problem (MVIP) associated with $F$ and $C$ consists in finding $x^{*} \in C$ and $u \in F\left(x^{*}\right)$ such that

$$
\left\langle u, y-x^{*}\right\rangle \geq 0, \forall y \in C
$$

MVIP (1) was first introduced and studied by Browder (1965) as an important generalization of the classical Variational Inequality Problem (VIP). The MVIP is also known to be a useful generalization of the class of multivalued complementarity problems (see Dong et al. 2017; Facchinei and Pang 2003; He et al. 2019), as well as constrained convex non-smooth optimization problems (see Dong et al. 2017; He et al. 2019; Rockafellar 1970). Therefore, problem (1) is quite general and provides a unified treatment for the study of a wide class of problems such as price equilibrium problems, oligopolistic market equilibrium problems, Nash equilibrium problems, fixed point problems for multivalued mappings, game theory, among others (see Attouch and Cabot 2019b; Brouwer 1912; Carey and Ge 2012; He et al. 2019; Nadler 1969; Oggioni et al. 2012; Raciti and Falsaperla 2007 and the references therein).

When $F$ is a singlevalued mapping in Eq. 1 (i.e., the case of the classical VIP), many methods have been designed by numerious authors for solving the VIP. These include, the gradient projection methods, extragradient methods (Korpelevich 1976), the subgradient extragradient methods (Censor et al. 2011), Tseng's method (Tseng 2000), among others (see, for example, Vuong 2019). We note that these methods for solving the classical VIP are not simply transformed to the case of MVIP since it is very difficult to handle the multivalued mapping associated with the MVIP. Thus, the methods for solving the MVIP are quite different. In 2014, Fang and Chen (2014) extended the subgradient extragradient method for solving the MVIP (1) in finite dimensional spaces. By employing the Procedure A below, they proposed the following Algorithm 1.1.

\section{Procedure A (Konnov 1998)}

Input: $\quad$ a point $x \in \mathbb{R}^{N}$.

Output: a point $R(x) \in C$, where $C:=\left\{x \in \mathbb{R}^{N} \mid g(x) \leq 0\right\}, g: \mathbb{R}^{N} \rightarrow \mathbb{R}$ is a convex function.

Step 1: $\quad$ set $n=0$ and $x_{n}=x$.

Step 2: if $g\left(x_{n}\right) \leq 0$, then stop and set $R(x)=x_{n}$. Otherwise, go to Step 3 .

Step 3: choose a point $w_{n} \in \partial g\left(x_{n}\right)$, where $\partial g(x)$ denotes the subdifferential of $g$ at $x$, set

$$
x_{n+1}=x_{n}-2 g\left(x_{n}\right) \frac{w_{n}}{\left\|w_{n}\right\|^{2}},
$$

and set $n:=n+1$ go back to Step 2 .

\section{Algorithm 1.1.}

Step 0: Choose $\bar{x}_{1} \in \mathbb{R}^{N}$ and two parameters $\gamma, \delta \in(0,1)$. Set $n=1$.

Step 1. Apply Procedure A with $x=\bar{x}_{n}$ and set $x_{n}=R\left(\bar{x}_{n}\right)$. 
Step 2. Choose $u_{n} \in F\left(x_{n}\right)$ and let $k_{n}$ be the smallest nonnegative integer satisfying $v_{n} \in F\left(P_{C}\left(x_{n}-\gamma^{k_{n}} u_{n}\right)\right)$,

$$
\gamma^{k_{n}}\left\|u_{n}-v_{n}\right\| \leq(1-\delta)\left\|x_{n}-P_{C}\left(x_{n}-\gamma^{k_{n}} u_{n}\right)\right\| .
$$

Set $\rho_{n}=\gamma^{k_{n}}$ and $z_{n}=P_{C}\left(x_{n}-\rho_{n} u_{n}\right)$. If $x_{n}=z_{n}$, stop.

Step 3. Compute $\bar{x}_{n+1}=P_{C_{n}}\left(x_{n}-\rho_{n} v_{n}\right)$, where $C_{n}=\left\{y \in \mathbb{R}^{N}:\left\langle x_{n}-\rho_{n} u_{n}-\right.\right.$ $\left.\left.z_{n}, y-z_{n}\right\rangle \leq 0\right\}$.

Let $n=n+1$ and return to Step 1 .

Inspired by Algorithm 1.1, Dong et al. (2017) proposed the following projection and contraction method for solving the MVIP (1).

\section{Algorithm 1.2.}

Step 0: Choose $\bar{x}_{1} \in \mathbb{R}^{N}$ and four parameters $\tau>0, \gamma, \delta \in(0,1)$ and $\alpha \in(0,2)$. Set $n=1$.

Step 1. Apply Procedure A with $x=\bar{x}_{n}$ and set $x_{n}=R\left(\bar{x}_{n}\right)$.

Step 2. Choose $u_{n} \in F\left(x_{n}\right)$ and find the smallest nonnegative integer $l_{k}$ such that $\rho_{n}=\tau \gamma^{l_{k}}$ and $v_{n} \in F\left(P_{C}\left(x_{n}-\rho_{n} u_{n}\right)\right)$, which satisfies

$$
\rho_{n}\left\|u_{n}-v_{n}\right\| \leq(1-\delta)\left\|x_{n}-P_{C}\left(x_{n}-\rho_{n} u_{n}\right)\right\| .
$$

Set $y_{n}=P_{C}\left(x_{n}-\rho_{n} u_{n}\right)$. If $x_{n}=y_{n}$, stop.

Step 3. Compute $\bar{x}_{n+1}=x_{n}-\alpha \beta_{n} d\left(x_{n}, y_{n}\right)$, where $d\left(x_{n}, y_{n}\right)-\rho_{n}\left(u_{n}-v_{n}\right)$, $\phi\left(x_{n}, y_{n}\right):=\left\langle x_{n}-y_{n}, d\left(x_{n}, y_{n}\right)\right\rangle$ and $\beta_{n}:=\frac{\phi\left(x_{n}, y_{n}\right)}{\left\|d\left(x_{n}, y_{n}\right)\right\|^{2}}$.

Let $n=n+1$ and return to Step 1 .

We comment that the Armijo-type linesearch procedures (2) and (3) of Algorithm 1.1 and Algorithm 1.2, respectively, involve the computation of projection onto $C$ multiple times in each linesearch. They also involve the evaluation of the multivalued mapping $F$ too many times in each search. To overcome some of these shortcomings, He et al. (2019), proposed the following projection-type method for solving MVIP (1):

\section{Algorithm 1.3.}

Step 0: Choose $x_{1} \in \mathbb{R}^{N}$ as an initial point and fix four parameters $\gamma, \sigma \in(0,1)$ and $0<\rho^{0} \leq \rho^{1}<\infty$. Set $C_{1}=\mathbb{R}^{N}, \bar{x}_{1}=x_{1}$, and $n=1$.

Step 1: Apply Procedure A to obtain $x_{n}=R\left(\bar{x}_{n}\right)$.

Step 2: Choose $u_{n} \in F\left(x_{n}\right)$ and $\rho_{n} \in\left[\rho^{0}, \rho^{1}\right]$. Set $y_{n}=P_{C}\left(x_{n}-\rho_{n} u_{n}\right)$. If $x_{n}=$ $y_{n}$, then stop. Otherwise, compute $z_{n}=\alpha_{n} y_{n}+\left(1-\alpha_{n}\right) x_{n}$ and choose the largest $\alpha \in\left\{\gamma^{0}, \gamma, \gamma^{2}, \gamma^{3}, \cdots\right\}$ such that there exists $w_{n} \in$ $F\left(z_{n}\right)$ satisfying

$$
\left\langle w_{n}, x_{n}-y_{n}\right\rangle \geq \sigma\left\langle u_{n}, x_{n}-y_{n}\right\rangle .
$$

Step 3: Taking a point $v_{n} \in F\left(y_{n}\right)$, set $d\left(x_{n}, y_{n}\right)=\left(x_{n}-y_{n}\right)-\rho_{n}\left(u_{n}-v_{n}\right)$ and compute $\bar{v}_{n}=x_{n}-\beta_{n} d\left(x_{n}, y_{n}\right)$, where $\beta_{n}=\frac{\phi\left(x_{n}, y_{n}\right)}{\left\|d\left(x_{n}, y_{n}\right)\right\|^{2}}, \quad \phi\left(x_{n}, y_{n}\right)=$ $\left\langle x_{n}-y_{n}, d\left(x_{n}, y_{n}\right)\right\rangle$. 
Step 4: Set $C_{n}=\left\{y \in \mathbb{R}^{N} \mid\left\langle w_{n}, y-z_{n}\right\rangle \leq 0\right\}$ for $n \geq 2$ and $C_{n}^{*}=\cap_{i=1}^{n} C_{i}$.

Compute $\bar{x}_{n+1}=P_{C_{n}^{*}}\left(\bar{v}_{n}\right)$.

If $\bar{x}_{n+1}=x_{n}$, then stop. Otherwise, let $n:=n+1$ and return Step 1 .

As observed in He et al. (2019, Section 4), Algorithm 1.1, Algorithm 1.2 and Algorithm 1.3 do not work well in some settings because of the presence of Procedure A in the iterative steps. Hence, the authors in He et al. (2019) proposed the following projection-type method without Procedure A for solving MVIP (1), which can be implemented in such settings.

\section{Algorithm 1.4.}

Step 0: Choose $x_{1} \in \mathbb{R}^{N}$ as an initial point and fix four parameters $\gamma, \sigma \in(0,1)$ and $0<\rho^{0} \leq \rho^{1}<\infty$. Set $C_{1}=\mathbb{R}^{N}$ and $n=1$.

Step 1: Choose $u_{n} \in F\left(x_{n}\right)$ and $\rho_{n} \in\left[\rho^{0}, \rho^{1}\right]$. Set $y_{n}=P_{C}\left(x_{n}-\rho_{n} u_{n}\right)$. If $x_{n}=y_{n}$, then stop. Otherwise, compute $z_{n}=\alpha_{n} y_{n}+\left(1-\alpha_{n}\right) x_{n}$ and choose the largest $\alpha \in\left\{\gamma^{0}, \gamma, \gamma^{2}, \gamma^{3}, \cdots\right\}$ such that there exists $w_{n} \in F\left(z_{n}\right)$ satisfying

$$
\left\langle w_{n}, x_{n}-y_{n}\right\rangle \geq \sigma\left\langle u_{n}, x_{n}-y_{n}\right\rangle
$$

Step 2: $\quad$ Taking a point $v_{n} \in F\left(y_{n}\right)$, set $d\left(x_{n}, y_{n}\right)=\left(x_{n}-y_{n}\right)-\rho_{n}\left(u_{n}-v_{n}\right)$ and compute $\bar{x}_{n}=x_{n}-\beta_{n} d\left(x_{n}, y_{n}\right)$, where $\beta_{n}=\frac{\phi\left(x_{n}, y_{n}\right)}{\left\|d\left(x_{n}, y_{n}\right)\right\|^{2}}, \quad \phi\left(x_{n}, y_{n}\right)=$ $\left\langle x_{n}-y_{n}, d\left(x_{n}, y_{n}\right)\right\rangle$.

Step 3: Set $C_{n}=\left\{y \in \mathbb{R}^{N} \mid\left\langle w_{n}, y-z_{n}\right\rangle \leq 0\right\}$ for $n \geq 2$ and $C_{n}^{*}=\cap_{i=1}^{n} C_{i}$.

Compute $x_{n+1}=P_{C \cap C_{n}^{*}}\left(\bar{x}_{n}\right)$.

If $x_{n+1}=x_{n}$, then stop. Otherwise, let $n:=n+1$ and return Step 1 .

Notice that the linesearch procedure in Algorithm 1.3 and Algorithm 1.4 involve the computation of the projection onto $C$ only one time in each search trial. Thus, Algorithm 1.3 and Algorithm 1.4 seem more efficient than Algorithm 1.1 and Algorithm 1.2. Moreover, He et al. (2019) showed numerically that their methods perform better than Algorithm 1.2 of Dong et al. (2017). However, Algorithm 1.3 and Algorithm 1.4 still involve the evaluation of the multivalued mapping at least 3 times in each iteration.

Recently, inertial type algorithms for solving optimization problems have become of great interest to numerous researchers. Since Polyak (1964) studied an inertial extrapolation process for solving the smooth convex minimization problems, there have been growing interests in the design and study of iterative methods with inertial term. For example, inertial forward-backward splitting methods (Attouch et al. 2000; Cholamjiak et al. 2018; Ochs et al. 2015), inertial Douglas-Rachford splitting method (Bot et al. 2015), inertial ADMM (Bot and Csetnek 2016), and inertial forwardbackward-forward method (Lorenz and Pock 2015). The inertial term is based upon a discrete analogue of a second order dissipative dynamical system (Attouch et al. 2000) and known for its efficiency in improving the convergence rate of iterative methods. The inertial type algorithms have been tested in the solution of certian number of problems (for example, imaging and data analysis problems, motion of a body 
in a potential field) and the tests show that they actually give remarkable speed-up when compared with corresponding algorithms without inertial term (see, for example, Attouch and Cabot 2019a; Attouch and Cabot 2019b; Attouch et al. 2000; Beck and Teboulle 2009; Bot and Csetnek 2016; Lorenz and Pock 2015; Ochs et al. 2015; Polyak 1964; Shehu and Cholamjiak 2019; Shehu et al. 2019; Shehu et al. 2019 and the references therein).

Inspired by this recent trend on inertial extrapolation type methods for solving optimization problems, our aim in this paper is to design some modifications of Algorithms 1.3 and 1.4, together with new inertial extrapolation techniques to solve problem (1). We present two inertial projection-type methods for solving MVIP (1) when the multivalued mapping $F$ is only assumed to be locally bounded without any monotonicity assumption. The first method uses a linesearch as in Algorithm 1.3 and Algorithm 1.4 while the second method uses a different linesearch procedure with the aim of minimizing the number of evaluation of the multivalued mapping $F$ in each search. Furthermore, the inertial techniques that we employ in this paper are quite different from the ones used in most papers (see for example Cholamjiak et al. 2018; Chuang 2017; Lorenz and Pock 2015; Mainge 2008; Moudafi and Oliny 2003; Ochs et al. 2015; Polyak 1964; Shehu and Cholamjiak 2019; Shehu et al. 2019; Shehu et al. 2019; Thong and Hieu 2018; Thong and Hieu 2017 and the references therein). Moreover, based on the weaker assumptions on the inertial factor in our methods, we derive several special cases of our methods. Finally, we provide some numerical implementations of our methods and compare them with the methods in He et al. (2019), in order to show the profits that we gain by introducing the inertial extrapolation steps.

We organize the rest of the paper as follows: We first recall some basic results in Section 2. Some discussions about our methods are given in Section 3. In Section 4, we investigate the convergence analysis of our first method. In Section 5, we analyze the convergence of our second method. In Section 6, we give some numerical experiments to support our theoretical findings. Then, we conclude with some final remarks in Section 7.

\section{Preliminaries}

The metric projection, denoted by $P_{C}$, is a map defined on $\mathbb{R}^{N}$ onto $C$ which assigns to each $x \in \mathbb{R}^{N}$, the unique point in $C$, denoted by $P_{C} x$ such that

$$
\left\|x-P_{C} x\right\|=\inf \{\|x-y\|: y \in C\} .
$$

It is well known that $P_{C}$ is nonexpansive, and characterized by the inequality

$$
\left\langle x-P_{C} x, y-P_{C} x\right\rangle \leq 0 \forall y \in C .
$$

Furthermore, the $P_{C}$ is known to possess the following property

$$
\left\|P_{C} x-x\right\|^{2} \leq\|x-y\|^{2}-\left\|P_{C} x-y\right\|^{2} \forall y \in C .
$$

It is also known that $P_{C}$ satisfies

$$
\left\langle x-z, x-P_{C} z\right\rangle \geq\left\|x-P_{C} z\right\|^{2}, \forall x \in C, z \in \mathbb{R}^{N} .
$$


For more information and properties of $P_{C}$, see Goebel and Reich (1984) and He (2006).

Definition 2.1 A multivalued mapping $F: C \rightrightarrows \mathbb{R}^{N}$ is said to be

- $\quad$ outer-semicontinuous at $x \in C$ if and only if the graph of $F$ is closed;

- inner-semicontinuous at $x \in C$ if for any sequence $\left\{x_{n}\right\}$ converging to $x$ and $y \in F(x)$, then there exists a sequence $\left\{y_{n}\right\}$ in $F\left(x_{n}\right)$ such that $\left\{y_{n}\right\}$ converges to $y$;

- continuous at $x \in C$ if it is both outer-semicontinuous and inner-semicontinuous at $x$;

- locally bounded on $C$ if for every $x \in C$, there exists a neighborhood $U$ of $x$ such that $F(U)$ is bounded, where $F(U)=\cup_{x \in U} F(x)$.

Definition 2.2 A multivalued mapping $F: C \rightrightarrows \mathbb{R}^{N}$ is said to be

- monotone on $C$ if for any $x, y \in C$,

$$
\langle u-v, x-y\rangle \geq 0, \forall u \in F(x), v \in F(y)
$$

- $\quad$ pseudomonotone on $C$ if for any $x, y \in C$,

$$
\text { there exists } u \in F(x):\langle u, y-x\rangle \geq 0 \text { implies } \forall v \in F(y):\langle v, y-x\rangle \geq 0 \text {; }
$$

- quasimonotone on $C$ if for any $x, y \in C$,

there exists $u \in F(x):\langle u, y-x\rangle>0$ implies $\forall v \in F(y):\langle v, y-x\rangle \geq 0$.

Proposition 2.3 (Rockafellar and Wets 2004) A multivalued mapping $F: C \rightrightarrows \mathbb{R}^{N}$ is said to be locally bounded if and only if for any bounded sequence $\left\{x_{n}\right\}$ with $u_{n} \in$ $F\left(x_{n}\right)$, the sequence $\left\{u_{n}\right\}$ is bounded.

Proposition 2.4 (He et al. 2019) Assume that the solution set of problem (1) $\Gamma$ is nonempty and that $F: C \rightrightarrows \mathbb{R}^{N}$ is continuous. If either

(i) $F$ is monotone or pseudomonotone on $C$;

(ii) $F$ is quasimonotone on $C$ and for any $x^{*} \in \Gamma$ with $u^{*} \in F\left(x^{*}\right)$ satisfying (1) such that

$$
\text { there exists } y^{*} \in C:\left\langle u^{*}, y^{*}-x^{*}\right\rangle \neq 0 \text {; }
$$

(iii) $F$ is quasimonotone on $C$ with int $C \neq \emptyset$ and $0 \notin F\left(x^{*}\right)$ for all $x^{*} \in \Gamma$.

Then,

$$
\left\langle u, y-x^{*}\right\rangle \geq 0 \forall y \in C, u \in F(y), x^{*} \in \Gamma
$$

Remark 2.5 We can see from Proposition 2.4 that condition (7) is a weaker condition than various monotonicity conditions. Thus, we shall assume for the rest of this paper, that the solution set of problem (1) $\Gamma$ is nonempty and that Eq. 7 is satisfied. 
Following Attouch and Cabot (2019a, pages 5, 10), we note that if $x_{n+1}=x_{n}+$ $\theta_{n}\left(x_{n}-x_{n-1}\right)$, then for all $n \geq 1$, we have that

$$
x_{n+1}-x_{n}=\left(\prod_{j=1}^{n} \theta_{j}\right)\left(x_{1}-x_{0}\right)
$$

which implies that

$$
x_{n}=x_{1}+\left(\sum_{j=1}^{n-1} \prod_{j=1}^{l} \theta_{j}\right)\left(x_{1}-x_{0}\right)
$$

Thus, $\left\{x_{n}\right\}$ converges if and only if $x_{1}=x_{0}$ or if $\sum_{l=1}^{\infty} \prod_{j=1}^{l} \theta_{j}<\infty$.

Therefore, we assume henceforth that

$$
\sum_{l=i}^{\infty}\left(\prod_{j=i}^{l} \theta_{j}\right)<\infty \quad \forall i \geq 1
$$

Then, we can define the sequence $\left\{t_{i}\right\}$ in $\mathbb{R}$ by

$$
t_{i}:=\sum_{l=i-1}^{\infty}\left(\prod_{j=i}^{l} \theta_{j}\right)=1+\sum_{l=i}^{\infty}\left(\prod_{j=i}^{l} \theta_{j}\right)
$$

with the convention $\prod_{j=i}^{i-1} \theta_{j}=1 \forall i \geq 1$.

Remark 2.6 Assumption (8) ensures that $\left\{t_{i}\right\}$ is well-defined in Eq. 9 and

$$
t_{i}=1+\theta_{i} t_{i+1}, \forall i \geq 1 .
$$

The following proposition provides a criterion for ensuring assumption (8). In fact, this condition makes it possible to cover the usual situations.

Proposition 2.7 (Attouch and Cabot 2019a, Proposition 3.1) Let $\left\{\theta_{n}\right\}$ be a sequence such that $\theta_{n} \in[0,1)$ for every $n \geq 1$. Assume that

$$
\lim _{n \rightarrow \infty}\left(\frac{1}{1-\theta_{n+1}}-\frac{1}{1-\theta_{n}}\right)=c
$$

for some $c \in[0,1)$. Then, we have

(i) Condition Eq. 8 holds, and $t_{n+1} \sim \frac{1}{(1-c)\left(1-\theta_{n}\right)}$ as $n \rightarrow \infty$.

(ii) The equivalence $1-\theta_{n} \sim 1-\theta_{n+1}$ holds true as $n \rightarrow \infty$. Hence, $t_{n+1} \sim t_{n+2}$ as $n \rightarrow \infty$. 
Remark 2.8 Example of a sequence satisfying the assumptions of Proposition 2.7 (therefore, satisfying assumption (8)) is $\theta_{n}=1-\frac{\bar{\theta}}{n}, \bar{\theta}>1$.

Clearly,

$$
\left(\frac{1}{1-\theta_{n+1}}-\frac{1}{1-\theta_{n}}\right)=\frac{1}{\bar{\theta}}(n+1)-\frac{1}{\bar{\theta}} n=\frac{1}{\bar{\theta}} .
$$

Hence,

$$
\lim _{n \rightarrow \infty}\left(\frac{1}{1-\theta_{n+1}}-\frac{1}{1-\theta_{n}}\right)=\frac{1}{\bar{\theta}} .
$$

Recall that the above example falls within the setting of Nesterov's extrapolation methods (for instance, see Attouch and Cabot 2019a; Beck and Teboulle 2009; Chambolle and Dossal 2015, Nesterov 1983).

The corresponding finite sum expression of $\left\{t_{i}\right\}$ is defined for $i, n \geq 1$, by

$$
t_{i, n}:= \begin{cases}\sum_{l=i-1}^{n-1}\left(\prod_{j=i}^{l} \theta_{j}\right)=1+\sum_{l=i}^{n-1}\left(\prod_{j=i}^{l} \theta_{j}\right), & i \leq n, \\ 0, & \text { otherwise. }\end{cases}
$$

In the same manner, we have that $\left\{t_{i, n}\right\}$ is well-defined and (see also Attouch and Cabot 2019a)

$$
t_{i, n}=1+\theta_{i} t_{i+1, n} \forall i \geq 1, n \geq i+1 .
$$

The sequences $\left\{t_{i}\right\}$ and $\left\{t_{i, n}\right\}$ are very crucial to our convergence analysis. In fact, their effect can be seen in the following lemma which also plays a crucial role in establishing our convergence results.

Lemma 2.9 (Attouch and Cabot 2019a, page 42, Lemma B.1). Let $\left\{a_{n}\right\},\left\{\theta_{n}\right\}$ and $\left\{w_{n}\right\}$ be sequences of real numbers satisfying

$$
a_{n+1} \leq \theta_{n} a_{n}+w_{n} \text { for every } n \geq 1 .
$$

Assume that $\theta_{n} \geq 0$ for every $n \geq 1$.

(a) For every $n \geq 1$, we have

$$
\sum_{i=1}^{n} a_{i} \leq t_{1, n} a_{1}+\sum_{i=1}^{n-1} t_{i+1, n} w_{i}
$$

where the double sequence $\left\{t_{i, n}\right\}$ is defined by Eq. 11 .

(b) Under Eq. 8, assume that the sequence $\left\{t_{i}\right\}$ defined by Eq. 9 satisfies $\sum_{i=1}^{\infty} t_{i+1}\left[w_{i}\right]_{+}<\infty$. Then, the series $\sum_{i \geq 1}\left[a_{i}\right]_{+}$is convergent, and

$$
\sum_{i=1}^{\infty}\left[a_{i}\right]_{+} \leq t_{1}\left[a_{1}\right]_{+}+\sum_{i=1}^{\infty} t_{i+1}\left[w_{i}\right]_{+}
$$

where $[t]_{+}:=\max \{t, 0\}$ for any $t \in \mathbb{R}$. 
The following lemmas will also be needed in our convergence analysis.

Lemma 2.10 (Facchinei and Pang 2003) A point $x^{*} \in \Gamma$ if and only if $x^{*}=P_{C}\left(x^{*}-\right.$ $\rho u)$ for some $u \in F\left(x^{*}\right)$ and $\rho>0$.

Lemma 2.11 (Attouch and Cabot 2019a, page 7, Lemma 2.1). Let $\left\{x_{n}\right\}$ be a sequence in $\mathbb{R}^{N}$, and let $\left\{\theta_{n}\right\}$ be a sequence of real numbers. Given $z \in \mathbb{R}^{N}$, define the sequence $\left\{\Gamma_{n}\right\}$ by $\Gamma_{n}:=\frac{1}{2}\left\|x_{n}-z\right\|^{2}$. Then

$$
\begin{aligned}
\Gamma_{n+1}-\Gamma_{n}-\theta_{n}\left(\Gamma_{n}-\Gamma_{n-1}\right)= & \frac{1}{2}\left(\theta_{n}+\theta_{n}^{2}\right)\left\|x_{n}-x_{n-1}\right\|^{2}+\left\langle x_{n+1}-y_{n}, x_{n+1}-z\right\rangle \\
& -\frac{1}{2}\left\|x_{n+1}-y_{n}\right\|^{2},
\end{aligned}
$$

where $y_{n}=x_{n}+\theta_{n}\left(x_{n}-x_{n-1}\right)$.

Lemma 2.12 The following is well-known:

$$
2\langle x, y\rangle=\|x\|^{2}+\|y\|^{2}-\|x-y\|^{2}=\|x+y\|^{2}-\|x\|^{2}-\|y\|^{2} \forall x, y \in \mathbb{R}^{N} .
$$

Lemma 2.13 (Konnov 1998) The number of iterations in Procedure A is finite and for any given $x \in \mathbb{R}^{N}$, it holds that

$$
\|R(x)-y\| \leq\|x-y\|, \quad \forall y \in C .
$$

\section{Proposed Methods}

In this section, we present our methods and discuss their features. We begin with the following assumptions under which we obtain our convergence results.

Assumption 3.1 Suppose that the following hold:

(a) The feasible set $C$ is nonempty, closed and convex subset of $\mathbb{R}^{N}$.

(b) $F: \mathbb{R}^{N} \rightrightarrows \mathbb{R}^{N}$ is locally bounded and continuous.

(c) $\Gamma$ is nonempty and satisfies condition (7).

(d) $\theta_{n} \in[0,1$ [ for all $n \geq 1$ and there exists $\varepsilon \in(0,1)$ such that for $n$ large enough, we have

$$
(1-\varepsilon)\left(1-\theta_{n-1}\right) \geq \theta_{n} t_{n+1}\left(1+\theta_{n}+\left[\theta_{n-1}-\theta_{n}\right]_{+}\right) .
$$

We now present some criteria that guarantee assumptions (8) and (14).

Proposition 3.1 Assume that $\left\{\theta_{n}\right\}$ is a nondecreasing sequence that satisfies $\theta_{n} \in$ $\left[0,1\left[\forall n \geq 1\right.\right.$ with $\lim _{n \rightarrow \infty} \theta_{n}=\theta$ such that the following condition holds:

$$
1-3 \theta>0
$$

Then assumptions (8) and (14) hold. 
Proof Observe that $\theta_{n} \leq \theta \quad \forall n \geq 1$. Thus, we have that assumption (8) is satisfied and $t_{n}=\frac{1}{1-\theta} \quad \forall n \geq 1$ (see Attouch and Cabot 2019a). Now, observe that $1-3 \theta>0$ implies that $(1-\theta)>\frac{\theta(1+\theta)}{1-\theta}$. This further implies that there exists $\epsilon \in(0,1)$ such that

$$
(1-\epsilon)(1-\theta) \geq \frac{\theta(1+\theta)}{1-\theta} .
$$

Since $\theta_{n} \leq \theta \forall n \geq 1$, we obtain from Eq. 16 that

$$
(1-\epsilon)\left(1-\theta_{n-1}\right) \geq \frac{\theta(1+\theta)}{1-\theta} \geq \theta_{n} t_{n+1}\left(1+\theta_{n}\right),
$$

for some $\epsilon \in(0,1)$. Since $\theta_{n-1} \leq \theta_{n} \forall n \geq 1$, we obtain that

$$
\theta_{n} t_{n+1}\left(1+\theta_{n}\right)=\theta_{n} t_{n+1}\left(1+\theta_{n}+\left[\theta_{n-1}-\theta_{n}\right]_{+}\right) .
$$

Combining this with Eq. 17, we get that the assumption (14) is satisfied.

Proposition 3.2 Suppose that $\theta_{n} \in[0,1)$ and there exists $c \in\left[0, \frac{1}{2}\right)$ such that

$$
\lim _{n \rightarrow \infty}\left(\frac{1}{1-\theta_{n+1}}-\frac{1}{1-\theta_{n}}\right)=c
$$

and

$$
\liminf _{n \rightarrow \infty}\left(1-\theta_{n}\right)^{2}>\limsup _{n \rightarrow \infty} \frac{\theta_{n}\left(1+\theta_{n}\right)}{1-2 c} .
$$

Then assumption (14) holds.

Proof From Eq. 19, we obtain that

$$
\liminf _{n \rightarrow \infty}\left(1-\theta_{n-1}\right)^{2}>\limsup _{n \rightarrow \infty} \frac{\theta_{n}\left(1+\theta_{n}\right)}{1-2 c} .
$$

Thus, there exists $\epsilon \in(0,1)$ sufficiently small enough such that

$$
\liminf _{n \rightarrow \infty}\left(1-\theta_{n-1}\right)^{2}>\limsup _{n \rightarrow \infty} \frac{\theta_{n}\left(1+\theta_{n}\right)}{1-2 c-\epsilon(1-c)}>\limsup _{n \rightarrow \infty} \frac{\theta_{n}\left(1+\theta_{n}\right)}{1-2 c} .
$$

This implies that

$$
\begin{aligned}
(1+o(1)) \theta_{n}\left(1+\theta_{n}\right) \quad & \leq[1-2 c-\epsilon(1-c)+o(1)]\left(1-\theta_{n-1}\right)^{2} \\
= & {[(1-\epsilon)(1-c)-(2 c-c+o(1))]\left(1-\theta_{n-1}\right)^{2} } \\
\leq & {\left[(1-\epsilon)(1-c)-\theta_{n}(c+o(1))\right]\left(1-\theta_{n-1}\right)^{2} }
\end{aligned}
$$

which implies that

$$
(1-\epsilon)(1-c)\left(1-\theta_{n-1}\right)^{2} \geq(1+o(1)) \theta_{n}\left(1+\theta_{n}+\left(1-\theta_{n-1}\right)^{2}+o\left(\left(1-\theta_{n-1}\right)^{2}\right)\right)
$$


Now, observe from Eq. 18 that

$$
\theta_{n-1}-\theta_{n}+c\left(1-\theta_{n-1}\right)\left(1-\theta_{n}\right)=o\left(\left(1-\theta_{n-1}\right)\left(1-\theta_{n}\right)\right),
$$

which implies from Proposition 2.7(ii) that

$$
\begin{aligned}
\theta_{n-1}-\theta_{n}= & -c\left(1-\theta_{n-1}\right)\left(1-\theta_{n}\right)+o\left(\left(1-\theta_{n-1}\right)\left(1-\theta_{n}\right)\right) \\
& =-c\left(1-\theta_{n-1}\right)^{2}+o\left(1-\theta_{n-1}\right)^{2} \text { as } n \rightarrow \infty .
\end{aligned}
$$

This implies that

$$
\begin{aligned}
&\left|\theta_{n-1}-\theta_{n}\right|=\left|-c\left(1-\theta_{n-1}\right)^{2}+o\left(1-\theta_{n-1}\right)^{2}\right| \\
& \leq c\left(1-\theta_{n-1}\right)^{2}+o\left(1-\theta_{n-1}\right)^{2} \text { as } n \rightarrow \infty
\end{aligned}
$$

Combining (22) and (23), we obtain that

$$
(1-\epsilon)(1-c)\left(1-\theta_{n-1}\right)^{2} \geq(1+o(1)) \theta_{n}\left(1+\theta_{n}+\left[\theta_{n-1}-\theta_{n}\right]_{+}\right) .
$$

By Proposition 2.7, we have that $t_{n+1} \sim t_{n} \sim \frac{1}{(1-c)\left(1-\theta_{n-1}\right)}$ as $n \rightarrow \infty$. Hence, Eq. 24 is equivalent to

$$
(1-\epsilon)(1-c)\left(1-\theta_{n-1}\right)^{2} \geq \frac{\theta_{n}}{(1-c)\left(1-\theta_{n-1}\right)} t_{n+1}\left(1+\theta_{n}+\left[\theta_{n-1}-\theta_{n}\right]_{+}\right),
$$

which implies that assumption (14) holds.

Remark 3.3 We mention that Proposition 3.1 and Proposition 3.2 provide some sufficient conditions for ensuring that assumptions (14) and (8) hold. That is, assumptions (14) and (8) are much more weaker conditions than the assumptions in both propositions. Note that similar conditions as in Propositions 3.1 and 3.2 have been used by other authors to ensure convergence of inertial methods (see Lorenz and Pock 2015; Thong and Hieu 2018; Thong and Hieu 2017 and the references therein). In fact, we shall see later that using the conditions in Proposition 3.1 and Proposition 3.2, we derive some corollaries of our results.

We now present the first method of this paper.

\section{Algorithm 3.2.}

Step 0: Choose the sequence $\left\{\theta_{n}\right\}$ in $[0,1)$ such that the condition from Eqs. 8 and 14 hold. Let $x_{1}, x_{0} \in \mathbb{R}^{N}$ be given arbitrary and fix $\gamma, \sigma \in(0,1), 0<$ Step 1. Set $\rho_{0} \leq \rho_{1}<\infty$. Set $C_{1}=\mathbb{R}^{N}$ and $n=1$.

$$
v_{n}=x_{n}+\theta_{n}\left(x_{n}-x_{n-1}\right)
$$

and choose $u_{n} \in F\left(v_{n}\right)$ and $\rho_{n} \in\left[\rho_{0}, \rho_{1}\right]$. Then, compute

$$
y_{n}=P_{C}\left(v_{n}-\rho_{n} u_{n}\right) \text {. If } v_{n}=y_{n} \text { : STOP. Otherwise, go to Step } 2 .
$$


Step 2. Compute

$$
z_{n}=\alpha_{n} y_{n}+\left(1-\alpha_{n}\right) v_{n}
$$

and choose the largest $\alpha \in\left\{\gamma, \gamma^{2}, \gamma^{3}, \ldots\right\}$ such that there exists a point $w_{n} \in F\left(z_{n}\right)$ satisfying

$$
\left\langle w_{n}, v_{n}-y_{n}\right\rangle \geq \sigma\left\langle u_{n}, v_{n}-y_{n}\right\rangle .
$$

Step 3. Set $C_{n}=\left\{y \in \mathbb{R}^{N}:\left\langle w_{n}, y-z_{n}\right\rangle \leq 0\right\}$ for $n \geq 2$ and $C_{n}^{*}=\cap_{i=1}^{n} C_{i}$. Then, compute

$$
x_{n+1}=P_{C_{n}^{*}}\left(v_{n}\right) .
$$

Set $n:=n+1$ and go back to Step 1 .

Lemma 3.4 Step 2 of Algorithm 3.2 is well-defined.

Proof Let $v \in C$ and $u \in F(v)$. Define $y:=P_{C}(v-\rho u), \rho>0$. If $v=y$, then by Lemma 2.10, we have that $v$ is a solution. Now, if $v \neq y$, then by Eq. 4,

$$
\langle u, v-y\rangle=\frac{1}{\rho}\langle y-(v-\rho u)+(v-y), v-y\rangle \geq \frac{1}{\rho}\langle v-y, v-y\rangle>0 .
$$

Now, suppose on the contrary that Step 2 is not well-defined, then we will have that, for any $\alpha>0$ and $w \in F(z)$ with $z=\alpha y+(1-\alpha) v$,

$$
\langle w, v-y\rangle<\sigma\langle u, v-y\rangle .
$$

In particular, for $\alpha_{n}=\frac{1}{n^{2}}$ with $z_{n}=\alpha_{n} y+\left(1-\alpha_{n}\right) v$, we have that $z_{n} \rightarrow v$ as $n \rightarrow \infty$. Since $F$ is continuous, it is inner-semicontinuous. Thus, there exists $w_{n} \in F\left(z_{n}\right)$ such that $w_{n} \rightarrow u$ with $u \in F(v)$. Taking $w$ as $w_{n}$ in Eq. 27, and taking limit as $n \rightarrow \infty$, we obtain that

$$
(1-\sigma)\langle u, v-y\rangle \leq 0,
$$

which contradicts (26). Hence, Step 2 of Algorithm 3.2 is well-defined.

Remark 3.5 Observe that Assumption 3.1 (c) ensures that Step 3 of Algorithm 3.2 is well-defined since $\Gamma \subset C_{n}^{*}$ and hence $C_{n}^{*} \neq \emptyset$ for all $n \geq 2$. Indeed, for $z \in \Gamma$, we obtain from Assumption 3.1 (c) that $\left\langle w_{n}, z-z_{n}\right\rangle \leq 0 \forall n \geq 2$. Thus, $z \in C_{n} \forall n \geq 2$, which follows that $z \in C_{n}^{*} \forall n \geq 2$.

In the following, we present another method with a new linesearch (different from Eq. 25) with the aim of minimizing the number of evaluation of the multivalued mapping $F$ in each search.

\section{Algorithm 3.3.}

Step 0: Choose the sequence $\left\{\theta_{n}\right\}$ such that the condition from Eqs. 8 and 14 hold. Let $x_{1}, x_{0} \in \mathbb{R}^{N}$ be given arbitrary and fix $\gamma, \sigma \in(0,1), 0<\rho_{0} \leq \rho_{1}<$ $\infty$. Set $C_{1}=\mathbb{R}^{N}$ and $n=1$. 
Step 1. Set

$$
v_{n}=x_{n}+\theta_{n}\left(x_{n}-x_{n-1}\right)
$$

and choose $u_{n} \in F\left(v_{n}\right)$ and $\rho_{n} \in\left[\rho_{0}, \rho_{1}\right]$. Then, compute

$$
y_{n}=P_{C}\left(v_{n}-\rho_{n} u_{n}\right) \text {. If } v_{n}=y_{n} \text { : STOP. Otherwise, go to Step } 2 .
$$

Step 2. Compute

$$
z_{n}=\alpha_{n} y_{n}+\left(1-\alpha_{n}\right) v_{n}
$$

and choose the largest $\alpha \in\left\{\gamma, \gamma^{2}, \gamma^{3}, \ldots\right\}$ such that there exists a point $w_{n} \in F\left(z_{n}\right)$ satisfying

$$
\left\langle w_{n}, v_{n}-y_{n}\right\rangle \geq \frac{\sigma}{2}\left\|v_{n}-y_{n}\right\|^{2} .
$$

Step 3. Set $C_{n}=\left\{y \in \mathbb{R}^{N}:\left\langle w_{n}, y-z_{n}\right\rangle \leq 0\right\}$ for $n \geq 2$ and $C_{n}^{*}=\cap_{i=1}^{n} C_{i}$. Then, compute

$$
x_{n+1}=P_{C_{n}^{*}}\left(v_{n}\right) .
$$

Set $n:=n+1$ and go back to Step $\mathbf{1}$.

Remark 3.6 (a) Observe that if we choose a point $u \in F(x)$ with $y:=P_{C}(x-\rho u)$, then, by setting $z=x-\rho u$ in Eq. 6 , we obtain that

$$
\langle u, x-y\rangle \geq \frac{\sigma}{2}\|x-y\|^{2} .
$$

Thus, using Eq. 28 and the continuity of $F$, we can see that Step 2 of Algorithm 3.3 is well-defined.

(b) Our Algorithm 3.2 and Algorithm 3.3 have fewer evaluations of multivalued mapping $F$ than Algorithm 1.3 and Algorithm 1.4.

\section{Convergence Analysis for Algorithm 3.2}

Lemma 4.1 Let $\left\{x_{n}\right\}$ be a sequence generated by Algorithm 3.2 and $\left\{\Gamma_{n}\right\}$ be defined by $\Gamma_{n}=\frac{1}{2}\left\|x_{n}-z\right\|^{2}$ for any $z \in \Gamma$. Then, under assumption (8) and Assumption $3.1(c),(d)$, we have that

$$
\begin{gathered}
\sum_{i=1}^{n-1}\left[t_{i+1, n}\left(\left(1-3 \theta_{i}\right)-\left(1-\theta_{i}\right)\right)+t_{i, n}\left(1-\theta_{i-1}\right)\right]\left\|x_{i}-x_{i-1}\right\|^{2} \\
\leq 2 t_{1}\left|\Gamma_{1}-\Gamma_{0}\right|+2 \Gamma_{0}+t_{1}\left(1-\theta_{0}\right)\left\|x_{1}-x_{0}\right\|^{2},
\end{gathered}
$$

where $\left\{t_{i, n}\right\}$ is defined in Eq. 11.

Proof First observe that

$$
\begin{aligned}
\left\|x_{n+1}-x_{n}\right\|^{2}= & \left\|x_{n+1}-2 x_{n}+x_{n-1}-\left(x_{n-1}-x_{n}\right)\right\|^{2} \\
= & \left\|x_{n+1}-2 x_{n}+x_{n-1}\right\|^{2}+\left\|x_{n-1}-x_{n}\right\|^{2} \\
& +2\left\langle x_{n+1}-2 x_{n}+x_{n-1}, x_{n}-x_{n-1}\right\rangle,
\end{aligned}
$$


which implies that

$2\left\langle x_{n+1}-2 x_{n}+x_{n-1}, x_{n}-x_{n-1}\right\rangle=\left\|x_{n+1}-x_{n}\right\|^{2}-\left\|x_{n+1}-2 x_{n}+x_{n-1}\right\|^{2}-\left\|x_{n-1}-x_{n}\right\|^{2}$.

Thus, we obtain that

$$
\begin{aligned}
\left\|x_{n+1}-v_{n}\right\|^{2}= & \left\|x_{n+1}-x_{n}-\left(x_{n}-x_{n-1}\right)+\left(1-\theta_{n}\right)\left(x_{n}-x_{n-1}\right)\right\|^{2} \\
= & \left\|x_{n+1}-2 x_{n}+x_{n-1}\right\|^{2}+\left(1-\theta_{n}\right)^{2}\left\|x_{n}-x_{n-1}\right\|^{2} \\
& +2\left(1-\theta_{n}\right)\left\langle x_{n+1}-2 x_{n}+x_{n-1}, x_{n}-x_{n-1}\right\rangle \\
= & \left\|x_{n+1}-2 x_{n}+x_{n-1}\right\|^{2}+\left(1-\theta_{n}\right)^{2}\left\|x_{n}-x_{n-1}\right\|^{2} \\
& +\left(1-\theta_{n}\right)\left[\left\|x_{n+1}-x_{n}\right\|^{2}-\left\|x_{n}-x_{n-1}\right\|^{2}-\left\|x_{n+1}-2 x_{n}+x_{n-1}\right\|^{2}\right] \\
= & \theta_{n}\left\|x_{n+1}-2 x_{n}+x_{n-1}\right\|^{2}+\left(1-\theta_{n}\right)^{2}\left\|x_{n}-x_{n-1}\right\|^{2} \\
& +\left(1-\theta_{n}\right)\left[\left\|x_{n+1}-x_{n}\right\|^{2}-\left\|x_{n}-x_{n-1}\right\|^{2}\right] \\
\geq & \left(1-\theta_{n}\right)^{2}\left\|x_{n}-x_{n-1}\right\|^{2}+\left(1-\theta_{n}\right)\left[\left\|x_{n+1}-x_{n}\right\|^{2}-\left\|x_{n}-x_{n-1}\right\|^{2}\right] .(29)
\end{aligned}
$$

Let $z \in \Gamma$, then by Remark 3.5, we have that $z \in C_{n}^{*}$. Thus, we obtain from Lemma 2.11 and Eq. 29 that

$$
\begin{aligned}
\Gamma_{n+1}-\Gamma_{n}-\theta_{n}\left(\Gamma_{n}-\Gamma_{n-1}\right)= & \frac{1}{2}\left(\theta_{n}+\theta_{n}^{2}\right)\left\|x_{n}-x_{n-1}\right\|^{2}+\left\langle x_{n+1}-v_{n}, x_{n+1}-z\right\rangle \\
& -\frac{1}{2}\left\|x_{n+1}-v_{n}\right\|^{2} \\
\leq & \frac{1}{2}\left(\theta_{n}+\theta_{n}^{2}\right)\left\|x_{n}-x_{n-1}\right\|^{2}-\frac{1}{2}\left\|x_{n+1}-v_{n}\right\|^{2} \\
\leq & \frac{1}{2}\left(\theta_{n}+\theta_{n}^{2}\right)\left\|x_{n}-x_{n-1}\right\|^{2}-\frac{1}{2}\left(1-\theta_{n}\right)^{2}\left\|x_{n}-x_{n-1}\right\|^{2} \\
& -\frac{1}{2}\left(1-\theta_{n}\right)\left[\left\|x_{n+1}-x_{n}\right\|^{2}-\left\|x_{n}-x_{n-1}\right\|^{2}\right] \\
= & \frac{1}{2}\left(3 \theta_{n}-1\right)\left\|x_{n}-x_{n-1}\right\|^{2}-\frac{1}{2}\left(1-\theta_{n}\right) \\
& \times\left[\left\|x_{n+1}-x_{n}\right\|^{2}-\left\|x_{n}-x_{n-1}\right\|^{2}\right],
\end{aligned}
$$

which implies from Lemma 2.9 (a) that

$$
\begin{aligned}
& \Gamma_{n}-\Gamma_{0}=\sum_{i=1}^{n}\left(\Gamma_{i}-\Gamma_{i-1}\right) \\
& \leq t_{1, n}\left(\Gamma_{1}-\Gamma_{0}\right)+\sum_{i=1}^{n-1} t_{i+1, n}\left[\frac{1}{2}\left(3 \theta_{i}-1\right)\left\|x_{i}-x_{i-1}\right\|^{2}-\frac{1}{2}\left(1-\theta_{i}\right)\right. \\
& \left.\quad \times\left(\left\|x_{i+1}-x_{i}\right\|^{2}-\left\|x_{i}-x_{i-1}\right\|^{2}\right)\right] .
\end{aligned}
$$


Notice that $t_{1, n} \leq t_{1}$. Thus, we obtain that

$$
\begin{aligned}
& \sum_{i=1}^{n-1} t_{i+1, n}\left[\left(1-3 \theta_{i}\right)\left\|x_{i}-x_{i-1}\right\|^{2}+\left(1-\theta_{i}\right)\left(\left\|x_{i+1}-x_{i}\right\|^{2}-\left\|x_{i}-x_{i-1}\right\|^{2}\right)\right] \\
\leq & 2 t_{1, n}\left(\Gamma_{1}-\Gamma_{0}\right)+2\left(\Gamma_{0}-\Gamma_{n}\right) \\
\leq & 2 t_{1}\left|\Gamma_{1}-\Gamma_{0}\right|+2 \Gamma_{0} .
\end{aligned}
$$

Now, observe that

$$
\begin{aligned}
& \sum_{i=1}^{n-1} t_{i+1, n}\left(1-\theta_{i}\right)\left(\left\|x_{i+1}-x_{i}\right\|^{2}-\left\|x_{i}-x_{i-1}\right\|^{2}\right) \\
= & \sum_{i=1}^{n-1}\left(t_{i, n}\left(1-\theta_{i-1}\right)-t_{i+1, n}\left(1-\theta_{i}\right)\right)\left\|x_{i}-x_{i-1}\right\|^{2} \\
& +t_{n, n}\left(1-\theta_{n-1}\right)\left\|x_{n}-x_{n-1}\right\|^{2}-t_{1, n}\left(1-\theta_{0}\right)\left\|x_{1}-x_{0}\right\|^{2} \\
\geq & \sum_{i=1}^{n-1}\left(t_{i, n}\left(1-\theta_{i-1}\right)-t_{i+1, n}\left(1-\theta_{i}\right)\right)\left\|x_{i}-x_{i-1}\right\|^{2}-t_{1}\left(1-\theta_{0}\right)\left\|x_{1}-x_{0}\right\|^{2} .
\end{aligned}
$$

Combining (31) and (32), we get that

$$
\begin{aligned}
& \sum_{i=1}^{n-1} t_{i+1, n}\left(1-3 \theta_{i}\right)\left\|x_{i}-x_{i-1}\right\|^{2}+\sum_{i=1}^{n-1}\left(t_{i, n}\left(1-\theta_{i-1}\right)-t_{i+1, n}\left(1-\theta_{i}\right)\right)\left\|x_{i}-x_{i-1}\right\|^{2} \\
\leq & 2 t_{1}\left|\Gamma_{1}-\Gamma_{0}\right|+2 \Gamma_{0}+t_{1}\left(1-\theta_{0}\right)\left\|x_{0}-x_{1}\right\|^{2} .
\end{aligned}
$$

That is,

$$
\begin{aligned}
& \sum_{i=1}^{n-1}\left[t_{i+1, n}\left(\left(1-3 \theta_{i}\right)-\left(1-\theta_{i}\right)\right)+t_{i, n}\left(1-\theta_{i-1}\right)\right]\left\|x_{i}-x_{i-1}\right\|^{2} \\
\leq & 2 t_{1}\left|\Gamma_{1}-\Gamma_{0}\right|+2 \Gamma_{0}+t_{1}\left(1-\theta_{0}\right)\left\|x_{0}-x_{1}\right\|^{2} .
\end{aligned}
$$

Lemma 4.2 Let $\left\{x_{n}\right\}$ be a sequence generated by Algorithm 3.2. Then, under assumption (8) and Assumption 3.1(c),(d), we have that $\sum_{n=1}^{\infty}\left(1-\theta_{n-1}\right)\left\|x_{n}-x_{n-1}\right\|^{2}<$ $\infty$ and $\sum_{n=1}^{\infty} \theta_{n} t_{n+1}\left\|x_{n}-x_{n-1}\right\|^{2}<\infty$ 
Proof From Eq. 12 and since $t_{i+1, n} \leq t_{i+1}$, we obtain

$$
\begin{aligned}
& t_{i+1, n}\left[\left(1-3 \theta_{i}\right)-\left(1-\theta_{i}\right)\right]+t_{i, n}\left(1-\theta_{i-1}\right) \\
= & t_{i+1, n}\left[\left(1-3 \theta_{i}\right)-\left(1-\theta_{i}\right)\right]+\left(1-\theta_{i-1}\right)+\theta_{i} t_{i+1, n}\left(1-\theta_{i-1}\right) \\
= & t_{i+1, n}\left[\left(1-3 \theta_{i}\right)-\left(1-\theta_{i}\right)+\theta_{i}\left(1-\theta_{i-1}\right)\right]+\left(1-\theta_{i-1}\right) \\
= & \left(1-\theta_{i-1}\right)-\theta_{i} t_{i+1, n}\left(1-\theta_{i-1}\right) \\
\geq & \left(1-\theta_{i-1}\right)-\theta_{i} t_{i+1}\left(1-\theta_{i-1}\right) \\
\geq & \left(1-\theta_{i-1}\right)-\theta_{i} t_{i+1}\left(1+\theta_{i}+\left[\theta_{i-1}-\theta_{i}\right]_{+}\right) .
\end{aligned}
$$

Using Eq. 34 in Lemma 4.1, we obtain that

$$
\begin{aligned}
& \sum_{i=1}^{n-1}\left(1-\theta_{i-1}\right)-\theta_{i} t_{i+1}\left(1+\theta_{i}+\left[\theta_{i-1}-\theta_{i}\right]_{+}\right)\left\|x_{i}-x_{i-1}\right\|^{2} \\
\leq & 2 t_{1}\left|\Gamma_{1}-\Gamma_{0}\right|+2 \Gamma_{0}+t_{1}\left(1-\theta_{0}\right)\left\|x_{0}-x_{1}\right\|^{2} .
\end{aligned}
$$

We may assume without loss of generality that assumption (14) holds for every $n \geq 1$. Then, we obtain that

$$
\sum_{i=1}^{n-1} \varepsilon\left(1-\theta_{i-1}\right)\left\|x_{i}-x_{i-1}\right\|^{2} \leq 2 t_{1}\left|\Gamma_{1}-\Gamma_{0}\right|+2 \Gamma_{0}+t_{1}\left(1-\theta_{0}\right)\left\|x_{0}-x_{1}\right\|^{2} .
$$

Now, taking limit as $n \rightarrow \infty$, we get that

$$
\sum_{i=1}^{\infty}\left(1-\theta_{i-1}\right)\left\|x_{i}-x_{i-1}\right\|^{2}<\infty
$$

Thus, the first conclusion of the lemma is established. To establish the second conclusion of the lemma, we employ assumption (14) again in Eq. 35 and obtain

$$
\sum_{i=1}^{\infty} \theta_{i} t_{i+1}\left\|x_{i}-x_{i-1}\right\|^{2}<\infty
$$

Lemma 4.3 Let $\left\{x_{n}\right\}$ be a sequence generated by Algorithm 3.2. Then, under assumption (8) and Assumption 3.1(c),(d), we have that

(a) $\lim _{n \rightarrow \infty}\left\|x_{n}-z\right\|$ exists for all $z \in \Gamma$.

(b) $\lim _{n \rightarrow \infty}\left\|v_{n}-x_{n+1}\right\|=0$.

Proof (a) From Eq. 30, we obtain that

$$
\begin{aligned}
\Gamma_{n+1}-\Gamma_{n} & \leq \theta_{n}\left(\Gamma_{n}-\Gamma_{n-1}\right)+\frac{1}{2}\left(\theta_{n}+\theta_{n}^{2}\right)\left\|x_{n}-x_{n-1}\right\|^{2}-\frac{1}{2}\left\|x_{n+1}-v_{n}\right\|^{2} \\
& \leq \theta_{n}\left(\Gamma_{n}-\Gamma_{n-1}\right)+\theta_{n}\left\|x_{n}-x_{n-1}\right\|^{2}-\frac{1}{2}\left\|x_{n+1}-v_{n}\right\|^{2} \\
& \leq \theta_{n}\left(\Gamma_{n}-\Gamma_{n-1}\right)+\theta_{n}\left\|x_{n}-x_{n-1}\right\|^{2} .
\end{aligned}
$$


Thus, from Lemma 4.2 and Lemma 2.9 (b), we obtain that $\sum_{n=1}^{\infty}\left[\Gamma_{n}-\Gamma_{n-1}\right]_{+}<$ $\infty$. This implies that $\lim _{n \rightarrow \infty} \Gamma_{n}=\lim _{n \rightarrow \infty} \frac{1}{2}\left\|x_{n}-z\right\|^{2}$ exists, which further gives that $\lim _{n \rightarrow \infty}\left\|x_{n}-z\right\|$ exists for all $z \in \Gamma$.

(b) Now, using Eq. 36 and Lemma 2.9 (a), we obtain that

$$
\begin{aligned}
\Gamma_{n}-\Gamma_{0} & =\sum_{i=1}^{n}\left(\Gamma_{i}-\Gamma_{i-1}\right) \\
& \leq t_{1, n}\left(\Gamma_{1}-\Gamma_{0}\right)+\sum_{i=1}^{n-1} t_{i+1, n}\left[\theta_{i}\left\|x_{i}-x_{i-1}\right\|^{2}-\frac{1}{2}\left\|x_{i+1}-v_{i}\right\|^{2}\right] .
\end{aligned}
$$

Since $t_{i+1, n} \leq t_{i+1}$, we obtain from Eq. 37 and Lemma 4.2 that

$$
\begin{aligned}
\sum_{i=1}^{n-1} t_{i+1, n}\left\|x_{i+1}-v_{i}\right\|^{2} & \leq 2 \Gamma_{0}+2 t_{1, n}\left(\Gamma_{1}-\Gamma_{0}\right)+2 \sum_{i=1}^{n-1} t_{i+1, n} \theta_{i}\left\|x_{i}-x_{i-1}\right\|^{2} \\
& \leq 2 \Gamma_{0}+2 t_{1}\left|\Gamma_{1}-\Gamma_{0}\right|+2 \sum_{i=1}^{\infty} t_{i+1} \theta_{i}\left\|x_{i}-x_{i-1}\right\|^{2}<\infty
\end{aligned}
$$

Since $t_{i+1, n}=0$ for $i \geq n$, letting $n$ tend to $\infty$, we obtain that

$$
\sum_{i=1}^{\infty} t_{i+1}\left\|x_{i+1}-v_{i}\right\|^{2}<\infty .
$$

Replacing $i$ with $n$ in Eq. 38 and since $t_{n} \geq 1$ for every $n \geq 1$, we obtain from Eq. 38 that $\sum_{n=1}^{\infty}\left\|x_{n+1}-v_{n}\right\|^{2}<\infty$. This implies that $\lim _{n \rightarrow \infty}\left\|v_{n}-x_{n+1}\right\|=0$.

Remark 4.4 The main role of assumption (14) is to guarantee the condition

$$
\sum_{n=1}^{\infty} t_{n+1} \theta_{n}\left\|x_{n}-x_{n-1}\right\|^{2}<\infty
$$

obtained in Lemma 4.2 above. Note that Lemma 4.3 holds true if we assume condition (39) directly. Moreover, if $\theta_{n} \in[0, \theta]$ for every $n \geq 1$, where $\theta \in[0,1)$, then $t_{n} \leq \frac{1}{(1-\theta)} \quad \forall n \geq 1$. Under this setting, we have that condition (39) is guaranteed by the condition

$$
\sum_{n=1}^{\infty} \theta_{n}\left\|x_{n}-x_{n-1}\right\|^{2}<\infty .
$$

In other words, if we assume that condition (40) holds for $\theta_{n} \in[0, \theta] \forall n \geq 1$, with $\theta \in[0,1)$, then Lemma 4.3 holds. This assumption has been used by numerous authors to ensure convergence of inertial methods (see, for example, Alvarez and Attouch 2001; Chuang 2017; Lorenz and Pock 2015; Mainge 2008; Moudafi and Oliny 2003 and the references therein). 
Furthermore, under the assumptions of Proposition 3.1, we obtain the following as corollaries of Lemma 4.2 and Lemma 4.3 respectively.

Corollary 4.5 Let $\left\{x_{n}\right\}$ be a sequence generated by Algorithm 3.2 such that Assumption 3.1(c) holds. Suppose that $\left\{\theta_{n}\right\}$ is a nondecreasing sequence that satisfies $\theta_{n} \in\left[0,1\left[\forall n \geq 1\right.\right.$ with $\lim _{n \rightarrow \infty} \theta_{n}=\theta$ such that $1-3 \theta>0$. Then, we have that $\sum_{n=1}^{\infty}\left(1-\theta_{n-1}\right)\left\|x_{n}-x_{n-1}\right\|^{2}<\infty$ and $\sum_{n=1}^{\infty} \theta_{n} t_{n+1}\left\|x_{n}-x_{n-1}\right\|^{2}<\infty$.

Proof By Proposition 3.1, we have that assumptions (8) and (14) hold. Hence, the proof follows from Lemma 4.2.

Corollary 4.6 Let $\left\{x_{n}\right\}$ be a sequence generated by Algorithm 3.2 such that Assumption 3.1(c) holds. Suppose that $\left\{\theta_{n}\right\}$ is a nondecreasing sequence that satisfies $\theta_{n} \in$ $\left[0,1\left[\forall n \geq 1\right.\right.$ with $\lim _{n \rightarrow \infty} \theta_{n}=\theta$ such that $1-3 \theta>0$. Then,

(a) $\lim _{n \rightarrow \infty}\left\|x_{n}-z\right\|$ exists for all $z \in \Gamma$.

(b) $\lim _{n \rightarrow \infty}\left\|v_{n}-x_{n+1}\right\|=0$.

Proof It is similar to the proof of Corollary 4.5.

Remark 4.7 Observe that Eq. 18 and Proposition 2.7 imply that condition (8) also holds in Proposition 3.2. Hence, by replacing assumptions (8) and (14) with the assumptions of Proposition 3.2 in Lemma 4.2 and Lemma 4.3, we also obtain corollaries of Lemma 4.2 and Lemma 4.3 in the same manner as Corollaries 4.5 and 4.6 respectively.

Remark 4.8 If we take the inertial factor $\theta_{n}$ to be a constant (that is $\theta_{n}=\theta \quad \forall n \geq 1$ ), then we obtain the following corollaries of Lemma 4.2 and Lemma 4.3.

Corollary 4.9 Let $\left\{x_{n}\right\}$ be a sequence generated by Algorithm 3.2 such that Assumption 3.1(c) holds. Suppose that $\theta_{n}=\theta \quad \forall n \geq 1$ with $\theta \in[0,1)$ such that

$$
(1-\theta)^{2}>\theta(1+\theta)
$$

Then, we have that $\sum_{n=1}^{\infty}(1-\theta)\left\|x_{n}-x_{n-1}\right\|^{2}<\infty$ and $\sum_{n=1}^{\infty} \frac{\theta}{1-\theta}\left\|x_{n}-x_{n-1}\right\|^{2}<\infty$. Consequently, we have $\sum_{n=1}^{\infty}\left\|x_{n}-x_{n-1}\right\|^{2}<\infty$. 
Proof Since $\theta_{n}=\theta \in[0,1)$, we obtain for $i \geq 1$ that $t_{i}=\sum_{l=i-1}^{\infty} \theta^{l-i+1}=\frac{1}{1-\theta}<$ $\infty$. Thus, we get that assumption (8) holds. Note also from Eq. 41 that there exists $\epsilon \in(0,1)$ such that

$$
(1-\epsilon)(1-\theta) \geq \frac{\theta(1+\theta)}{1-\theta},
$$

which is equivalent to condition (14) since $\theta_{n}=\theta \quad \forall n \geq 1$. Hence, all the assumptions of Lemma 4.2 are satisfied. Thus, the rest of the proof follows from Lemma 4.2.

Corollary 4.10 Let $\left\{x_{n}\right\}$ be a sequence generated by Algorithm 3.2 such that Assumption 3.1(c) holds. Suppose that $\theta_{n}=\theta \quad \forall n \geq 1$ with $\theta \in[0,1)$ such that $(1-\theta)^{2}>\theta(1+\theta)$. Then,

(a) $\lim _{n \rightarrow \infty}\left\|x_{n}-z\right\|$ exists for all $z \in \Gamma$.

(b) $\lim _{n \rightarrow \infty}\left\|v_{n}-x_{n+1}\right\|=0$.

Proof The proof is similar to the proof of Corollary 4.9.

We now return to a very important result for our convergence analysis, whose proof rely on the linesearch given in Algorithm 3.2.

Lemma 4.11 Let assumption (8) and Assumption 3.1 hold, and let the sequence $\left\{x_{n}\right\}$ be generated by Algorithm 3.2. Then, $\lim _{n \rightarrow \infty} \alpha_{n}\left\|y_{n}-v_{n}\right\|^{2}=0$. Moreover, if there exists a subsequence $\left\{x_{n_{k}}\right\}$ of $\left\{x_{n}\right\}$ such that $\left\{x_{n_{k}}\right\}$ converges to $x^{*}$ and $x^{*} \notin \Gamma$, then

(a) $\liminf _{k \rightarrow \infty} \alpha_{n_{k}}>0$;

(b) $\lim _{k \rightarrow \infty}\left\|v_{n_{k}}-y_{n_{k}}\right\|=0$.

Proof From Eq. 4, Step 1, Step 2 and the fact that $x_{n+1} \in C_{n}^{*}$, we obtain that

$$
\begin{aligned}
\alpha_{n}\left\|v_{n}-y_{n}\right\|^{2} & =\alpha_{n}\left\langle v_{n}-y_{n}, v_{n}-y_{n}\right\rangle \\
& \leq \alpha_{n}\left\langle v_{n}-y_{n}, v_{n}-y_{n}\right\rangle+\alpha_{n}\left\langle y_{n}-v_{n}+\rho_{n} u_{n}, v_{n}-y_{n}\right\rangle \\
& =\alpha_{n} \rho_{n}\left\langle u_{n}, v_{n}-y_{n}\right\rangle \\
& \leq \frac{\alpha_{n} \rho_{n}}{\sigma}\left\langle w_{n}, v_{n}-y_{n}\right\rangle \\
& =\frac{\rho_{n}}{\sigma}\left\langle w_{n}, v_{n}-z_{n}\right\rangle \\
& \leq \frac{\rho_{n}}{\sigma}\left(\left\langle w_{n}, v_{n}-x_{n+1}\right\rangle+\left\langle w_{n}, x_{n+1}-z_{n}\right\rangle\right) \\
& \leq \frac{\rho_{n}}{\sigma}\left\|w_{n}\right\|\left\|v_{n}-x_{n+1}\right\| .
\end{aligned}
$$


Since by Lemma 4.3, $\left\{x_{n}\right\}$ is bounded, we have that $\left\{z_{n}\right\}$ is also bounded. Moreover, since $F$ is locally bounded, we obtain from Proposition 2.3 that $\left\{w_{n}\right\}$ is bounded. Using this and the boundedness of $\left\{\rho_{n}\right\}$, we obtain from Eq. 42 and Lemma 4.3 that

$$
\lim _{n \rightarrow \infty} \alpha_{n}\left\|y_{n}-v_{n}\right\|^{2}=0 \text {. }
$$

(a) By Step 2, we have that $\left\{\alpha_{n}\right\} \subset(0,1)$ is bounded. Thus, there exists a subsequence $\left\{\alpha_{n_{k}}\right\}$ of $\left\{\alpha_{n}\right\}$ such that $\liminf _{k \rightarrow \infty} \alpha_{n_{k}} \geq 0$.

In fact, we claim that $\liminf _{k \rightarrow \infty} \alpha_{n_{k}}>0$. Suppose on the contrary that $\liminf _{k \rightarrow \infty} \alpha_{n_{k}}=$ 0 . Then, without loss of generality, we can choose a subsequence of $\left\{\alpha_{n_{k}}\right\}$ still denoted by $\left\{\alpha_{n_{k}}\right\}$ such that $\lim _{k \rightarrow \infty} \alpha_{n_{k}}=0$.

Now, define $\bar{\alpha}_{n_{k}}:=\frac{\alpha_{n_{k}}}{\gamma}, \bar{z}_{n_{k}}:=\bar{\alpha}_{n_{k}} y_{n_{k}}+\left(1-\bar{\alpha}_{n_{k}}\right) v_{n_{k}}$. Then, by the boundedness of $\left\{y_{n_{k}}-v_{n_{k}}\right\}$ and since $\alpha_{n_{k}} \rightarrow 0$ as $k \rightarrow \infty$, we obtain that

$$
\lim _{k \rightarrow \infty}\left\|\bar{z}_{n_{k}}-v_{n_{k}}\right\|=0 .
$$

Also, by Lemma 4.2, we obtain that $\lim _{k \rightarrow \infty}\left\|x_{n_{k}}-v_{n_{k}}\right\|=\lim _{k \rightarrow \infty} \theta_{n_{k}}\left\|x_{n_{k}}-x_{n_{k}-1}\right\|=0$. Thus, since $x_{n_{k}} \rightarrow x^{*}$, we have that $v_{n_{k}} \rightarrow x^{*}$. Using Assumption $3.1(\mathrm{~b})$, the boundedness of $\left\{v_{n_{k}}\right\}$ and Proposition 2.3, we obtain that $\left\{u_{n_{k}}\right\}$ is also bounded. Thus, we can choose a subsequence of $\left\{u_{n_{k}}\right\}$ still denoted by $\left\{u_{n_{k}}\right\}$ such that $u_{n_{k}} \rightarrow \bar{u}$. Since $F$ is continuous, it is outer-semicontinuous. Hence, $\bar{u} \in F\left(x^{*}\right)$. We also assume without loss of generality that $\rho_{n_{k}} \rightarrow \rho \in\left[\rho_{0}, \rho_{1}\right]$. Therefore, we obtain from the continuity of $P_{C}$ that $y_{n_{k}} \rightarrow y^{*}$ as $k \rightarrow \infty$, where $y^{*}=P_{C}\left(x^{*}-\rho \bar{u}\right)$.

Again, from Eq. 44, we obtain that $\bar{z}_{n_{k}} \rightarrow x^{*}$. Since $F$ is inner-semicontinuous and $\bar{u} \in F\left(x^{*}\right)$, we can choose a subsequence $w_{n_{k}} \in F\left(\bar{z}_{n_{k}}\right)$ such that $\bar{w}_{n_{k}} \rightarrow \bar{u}$.

Now, from the definition of $\bar{z}_{n_{k}}$ and Step 2, we obtain that

$$
\left\langle\bar{w}_{n_{k}}, v_{n_{k}}-y_{n_{k}}\right\rangle<\sigma\left\langle u_{n_{k}}, v_{n_{k}}-y_{n_{k}}\right\rangle .
$$

Thus, taking limit as $k \rightarrow \infty$, we obtain that

$$
\left\langle\bar{u}, x^{*}-y^{*}\right\rangle \leq 0 .
$$

On the hand, since $x^{*} \notin \Gamma$, we have from Lemma 2.10 that $x^{*} \neq y^{*}$. Hence, we get

$$
\left\langle\bar{u}, x^{*}-y^{*}\right\rangle=\frac{1}{\rho}\left\langle y^{*}-\left(x^{*}-\rho \bar{u}\right)+\left(x^{*}-y^{*}\right), x^{*}-y^{*}\right\rangle>\frac{1}{\rho}\left\langle x^{*}-y^{*}, x^{*}-y^{*}\right\rangle>0,
$$

which is a contradiction to Eq. 46. Therefore, $\liminf _{k \rightarrow \infty} \alpha_{n_{k}}>0$.

(b) From (a), we have that $\liminf _{k \rightarrow \infty} \alpha_{n_{k}}>0$. Thus, we obtain from Eq. 43 that

$$
\begin{gathered}
0 \leq \limsup _{k \rightarrow \infty}\left\|v_{n_{k}}-y_{n_{k}}\right\|^{2} \leq \limsup _{k \rightarrow \infty}\left(\alpha_{n_{k}}\left\|v_{n_{k}}-y_{n_{k}}\right\|^{2}\right)\left(\limsup _{k \rightarrow \infty} \frac{1}{\alpha_{n_{k}}}\right) \\
=\left(\limsup _{k \rightarrow \infty} \alpha_{n_{k}}\left\|v_{n_{k}}-y_{n_{k}}\right\|^{2}\right)\left(\frac{1}{\liminf _{k \rightarrow \infty} \alpha_{n_{k}}}\right) \\
=0 .
\end{gathered}
$$


Therefore, we obtain that

$$
\lim _{k \rightarrow \infty}\left\|v_{n_{k}}-y_{n_{k}}\right\|=0
$$

Now, we are in position to give the main theorem of this section.

Theorem 4.12 Let $\left\{x_{n}\right\}$ be a sequence generated by Algorithm 3.2. Then, under assumption (8) and Assumption 3.1, we have that $\left\{x_{n}\right\}$ converges to an element of $\Gamma$.

Proof By Lemma 4.3, $\left\{x_{n}\right\}$ is bounded. Thus, there exists a subsequence $\left\{x_{n_{k}}\right\}$ of $\left\{x_{n}\right\}$ such that $\left\{x_{n_{k}}\right\}$ converges to some point $x^{*}$. Also, we have that

$$
\left\|v_{n_{k}}-x_{n_{k}}\right\|=\theta_{n_{k}}\left\|x_{n_{k}}-x_{n_{k}-1}\right\| \rightarrow 0, \text { as } k \rightarrow \infty
$$

We now claim that $x^{*} \in \Gamma$.

Suppose on the contrary that $x^{*} \notin \Gamma$. Then, it follows from Lemma 4.11 (b) and Eq. 48 that

$$
\lim _{k \rightarrow \infty} y_{n_{k}}=\lim _{k \rightarrow \infty} P_{C}\left(v_{n_{k}}-\rho_{n_{k}} u_{n_{k}}\right)=\lim _{k \rightarrow \infty} x_{n_{k}}=x^{*}
$$

Now, without loss of generality, we may assume that $\rho_{n_{k}} \rightarrow \rho^{*}$ and $u_{n_{k}} \rightarrow u^{*}$. Since $F$ is continuous, it is outer-semicontinuous. Thus, we obtain that $u^{*} \in F\left(x^{*}\right)$. Therefore, we obtain from Eq. 49 that

$$
P_{C}\left(x^{*}-\rho^{*} u^{*}\right)=x^{*} .
$$

It then follows from Lemma 2.10 that $x^{*} \in \Gamma$, which is a contraction. Hence, our claim holds.

We now show that $\left\{x_{n}\right\}$ converges to $x^{*}$.

Replacing $z$ by $x^{*}$ in Lemma 4.3, we obtain that $\lim _{n \rightarrow \infty}\left\|x_{n}-x^{*}\right\|^{2}$ exists. Since $x^{*}$ is an accumulation point of $\left\{x_{n}\right\}$, we obtain that $\left\{x_{n}\right\}$ converges to $x^{*}$.

Remark 4.13 In view of Corollaries 4.5-4.10, we can obtain various corollaries of Theorem 4.12. Furthermore, in the case that $\theta_{n}=0$ for all $n \geq 1$, assumptions (8) and (14) are automatically satisfied. Moreover, we have in this case that $t_{n}=1$ for all $n \geq 1$. Hence, we can employ Procedure A (see page 1) to obtain similar result as in He et al. (2019, Theorem 3.1).

\section{Algorithm 4.1.}

Step 0: Let $x_{1} \in \mathbb{R}^{N}$ be given arbitrary and fix $\gamma, \sigma \in(0,1), 0<\rho_{0} \leq \rho_{1}<\infty$. Set $C_{1}=\mathbb{R}^{N}, \bar{x}_{1}=x_{1}$ and $n=1$.

Step 1. Apply Procedure A to obtain $x_{n}=R\left(\bar{x}_{n}\right)$.

Step 2. Choose $u_{n} \in F\left(x_{n}\right)$ and $\rho_{n} \in\left[\rho_{0}, \rho_{1}\right]$. Then, compute

$$
y_{n}=P_{C}\left(x_{n}-\rho_{n} u_{n}\right) \text {. If } x_{n}=y_{n} \text { : STOP. Otherwise, go to Step } 2 .
$$


Step 3. Compute

$$
z_{n}=\alpha_{n} y_{n}+\left(1-\alpha_{n}\right) x_{n}
$$

and choose the largest $\alpha \in\left\{\gamma, \gamma^{2}, \gamma^{3}, \ldots\right\}$ such that there exists a point $w_{n} \in F\left(z_{n}\right)$ satisfying

$$
\left\langle w_{n}, x_{n}-y_{n}\right\rangle \geq \sigma\left\langle u_{n}, x_{n}-y_{n}\right\rangle .
$$

Step 4. Set $C_{n}=\left\{y \in \mathbb{R}^{N}:\left\langle w_{n}, y-z_{n}\right\rangle \leq 0\right\}$ for $n \geq 2$ and $C_{n}^{*}=\cap_{i=1}^{n} C_{i}$. Then, compute

$$
\bar{x}_{n+1}=P_{C_{n}^{*}}\left(x_{n}\right) .
$$

If $\bar{x}_{n+1}=x_{n}$, then stop. Otherwise, let $n=n+1$ and return to Step 1 .

Corollary 4.14 (see for example, He et al. (2019, Theorem 3.1)) Let $\left\{x_{n}\right\}$ be a sequence generated by Algorithm 4.1 such that the following assumptions hold:

(a) The set $C$ is described as in procedure $A$ (see page 1).

(b) $F: C \rightrightarrows \mathbb{R}^{N}$ is locally bounded and continuous.

(c) $\Gamma$ is nonempty and satisfies condition (7).

Then, we have that $\left\{x_{n}\right\}$ converges to an element of $\Gamma$.

Proof It follows carefully from Lemma 2.13 and Theorem 4.12.

Remark 4.15 Under the settings of Remark 4.13, we can obtain in general, similar result as in He et al. (2019, Theorem 3.2) without Procedure A.

\section{Algorithm 4.2.}

Step 0: Let $x_{1} \in C$ be given arbitrary and fix $\gamma, \sigma \in(0,1), 0<\rho_{0} \leq \rho_{1}<\infty$. Set $C_{1}=\mathbb{R}^{N}$ and $n=1$.

Step 1. Choose $u_{n} \in F\left(x_{n}\right)$ and $\rho_{n} \in\left[\rho_{0}, \rho_{1}\right]$. Then, compute

Step 2. Compute

$$
y_{n}=P_{C}\left(x_{n}-\rho_{n} u_{n}\right) \text {. If } x_{n}=y_{n} \text { : STOP. Otherwise, go to Step } 2 .
$$

$$
z_{n}=\alpha_{n} y_{n}+\left(1-\alpha_{n}\right) x_{n}
$$

and choose the largest $\alpha \in\left\{\gamma, \gamma^{2}, \gamma^{3}, \ldots\right\}$ such that there exists a point $w_{n} \in F\left(z_{n}\right)$ satisfying

$$
\left\langle w_{n}, x_{n}-y_{n}\right\rangle \geq \sigma\left\langle u_{n}, x_{n}-y_{n}\right\rangle .
$$

Step 3. Set $C_{n}=\left\{y \in \mathbb{R}^{N}:\left\langle w_{n}, y-z_{n}\right\rangle \leq 0\right\}$ for $n \geq 2$ and $C_{n}^{*}=\cap_{i=1}^{n} C_{i}$. Then, compute

$$
x_{n+1}=P_{C} \cap C_{n}^{*}\left(x_{n}\right) .
$$

If $x_{n+1}=x_{n}$, then stop. Otherwise, let $n=n+1$ and return to Step 1 . 
Corollary 4.16 (see, for example, He et al. (2019, Theorem 3.2)) Let $\left\{x_{n}\right\}$ be a sequence generated by Algorithm 4.2 such that the following assumptions hold:

(a) The feasible set $C$ is a nonempty closed and convex subset of $\mathbb{R}^{N}$.

(b) $F: C \rightrightarrows \mathbb{R}^{N}$ is locally bounded and continuous.

(c) $\Gamma$ is nonempty and satisfies condition (7).

Then, we have that $\left\{x_{n}\right\}$ converges to an element of $\Gamma$.

Proof It follows directly from Corollary 4.14 .

\section{Convergence Analysis for Algorithm 3.3}

Remark 5.1 Notice that Step 2 (the linesearch procedure) of Algorithm 3.2 is not utilized in the proof of Lemma 4.1-Lemma 4.3. Thus, Lemma 4.1-Lemma 4.3 hold automatically if $\left\{x_{n}\right\}$ is generated by Algorithm 3.3. Therefore, we only need to prove the version of Lemma 4.11 and Theorem 4.12 corresponding to Algorithm 3.3 in this section.

Lemma 5.2 Let the sequence $\left\{x_{n}\right\}$ be generated by Algorithm 3.3 such that assumption (8) and Assumption 3.1 are satisfied. Then, we have

(a) $\lim _{n \rightarrow \infty} \alpha_{n}\left\|y_{n}-v_{n}\right\|^{2}=0$.

(b) If there exists a subsequence $\left\{x_{n_{k}}\right\}$ of $\left\{x_{n}\right\}$ such that $\left\{x_{n_{k}}\right\}$ converges to $x^{*}$, then $\lim _{k \rightarrow \infty}\left\|v_{n_{k}}-y_{n_{k}}\right\|=0$

Proof (a) From Eq. 4, Step 2 and the fact that $x_{n+1} \in C_{n}^{*}$, we obtain that

$$
\begin{aligned}
\alpha_{n}\left\|v_{n}-y_{n}\right\|^{2} & \leq \frac{2 \alpha_{n}}{\sigma}\left\langle w_{n}, v_{n}-y_{n}\right\rangle \\
& \leq \frac{2}{\sigma}\left\langle w_{n}, v_{n}-z_{n}\right\rangle \\
& \leq \frac{2}{\sigma}\left(\left\langle w_{n}, v_{n}-x_{n+1}\right\rangle+\left\langle w_{n}, x_{n+1}-z_{n}\right\rangle\right) \\
& \leq \frac{2}{\sigma}\left\|w_{n}\right\|\left\|v_{n}-x_{n+1}\right\| .
\end{aligned}
$$

Since $\left\{z_{n}\right\}$ is bounded and $F$ is locally bounded, we obtain from Proposition 2.3 that $\left\{w_{n}\right\}$ is also bounded. Thus, we obtain from Eq. 52 and Lemma 4.3 that

$$
\lim _{n \rightarrow \infty} \alpha_{n}\left\|y_{n}-v_{n}\right\|^{2}=0
$$

(b) Since $\left\{\alpha_{n}\right\} \subset(0,1)$ is bounded, we have that $\liminf _{n \rightarrow \infty} \alpha_{n} \geq 0$. 
We now consider two possible cases:

Case 1: Suppose that $\liminf _{n \rightarrow \infty} \alpha_{n}=0$. Then, we can choose a subsequence of $\left\{\alpha_{n}\right\}$ denoted by $\left\{\alpha_{n_{k}}\right\}$ such that $\lim _{k \rightarrow \infty} \alpha_{n_{k}}=0$ and

$$
\lim _{k \rightarrow \infty}\left\|v_{n_{k}}-y_{n_{k}}\right\|=t \geq 0
$$

Now, define $\bar{\alpha}_{n_{k}}:=\frac{\alpha_{n_{k}}}{\gamma}$. Then, $\bar{z}_{n_{k}}:=\bar{\alpha}_{n_{k}} y_{n_{k}}+\left(1-\bar{\alpha}_{n_{k}}\right) v_{n_{k}}$. Since $\alpha_{n_{k}} \rightarrow 0$ as $k \rightarrow \infty$, we obtain that $\bar{\alpha}_{n_{k}} \rightarrow 0$ as $k \rightarrow \infty$. Hence,

$$
\lim _{k \rightarrow \infty}\left\|\bar{z}_{n_{k}}-v_{n_{k}}\right\|=0
$$

Now, from the definition of $\bar{z}_{n_{k}}$ and Step 2, we obtain that

$$
\left\langle\bar{w}_{n_{k}}, v_{n_{k}}-y_{n_{k}}\right\rangle<\frac{\sigma}{2}\left\|v_{n_{k}}-y_{n_{k}}\right\|^{2},
$$

which implies that

$$
2\left\langle\bar{w}_{n_{k}}-u_{n_{k}}, v_{n_{k}}-y_{n_{k}}\right\rangle+2\left\langle u_{n_{k}}, v_{n_{k}}-y_{n_{k}}\right\rangle<\sigma\left\|v_{n_{k}}-y_{n_{k}}\right\|^{2} .
$$

Now, set $s_{n_{k}}:=v_{n_{k}}-\rho_{n_{k}} u_{n_{k}}$. Then, Eq. 56 becomes

$$
2\left\langle\bar{w}_{n_{k}}-u_{n_{k}}, v_{n_{k}}-y_{n_{k}}\right\rangle+\frac{2}{\rho_{n_{k}}}\left\langle v_{n_{k}}-s_{n_{k}}, v_{n_{k}}-y_{n_{k}}\right\rangle<\sigma\left\|v_{n_{k}}-y_{n_{k}}\right\|^{2},
$$

which implies that

$2\left\langle\bar{w}_{n_{k}}-u_{n_{k}}, v_{n_{k}}-y_{n_{k}}\right\rangle+\frac{1}{\rho_{n_{k}}}\left(\left\|v_{n_{k}}-y_{n_{k}}\right\|^{2}+\left\|s_{n_{k}}-v_{n_{k}}\right\|^{2}-\left\|s_{n_{k}}-y_{n_{k}}\right\|^{2}\right)<\sigma\left\|v_{n_{k}}-y_{n_{k}}\right\|^{2}$.

That is,

$$
\frac{1}{\rho_{n_{k}}}\left(\left\|s_{n_{k}}-v_{n_{k}}\right\|^{2}-\left\|s_{n_{k}}-y_{n_{k}}\right\|^{2}\right)<\left(\sigma-\frac{1}{\rho_{n_{k}}}\right)\left\|v_{n_{k}}-y_{n_{k}}\right\|^{2}-2\left\langle\bar{w}_{n_{k}}-u_{n_{k}}, v_{n_{k}}-y_{n_{k}}\right\rangle .
$$

Now, by Lemma 4.2, we obtain that $\lim _{k \rightarrow \infty}\left\|x_{n_{k}}-v_{n_{k}}\right\|=0$. Thus, since $x_{n_{k}} \rightarrow x^{*}$, we have that $v_{n_{k}} \rightarrow x^{*}$. Using Assumption $3.1(\mathrm{~b})$, the boundedness of $\left\{v_{n_{k}}\right\}$ and Proposition 2.3, we obtain that $\left\{u_{n_{k}}\right\}$ is also bounded. Thus, we can choose a subsequence of $\left\{u_{n_{k}}\right\}$ still denoted by $\left\{u_{n_{k}}\right\}$ such that $u_{n_{k}} \rightarrow \bar{u}$. Since $F$ is continuous, it is outer-semicontinuous. Hence, $\bar{u} \in F\left(x^{*}\right)$. We also assume without loss of generality that $\rho_{n_{k}} \rightarrow \rho \in\left[\rho_{0}, \rho_{1}\right] \subset\left[\rho_{0}, \frac{1}{\sigma}\right)$. Again, from Eq. 55, we obtain that $\bar{z}_{n_{k}} \rightarrow x^{*}$. Since $F$ is inner-semicontinuous and $\bar{u} \in F\left(x^{*}\right)$, we can choose a subsequence $w_{n_{k}} \in F\left(\bar{z}_{n_{k}}\right)$ such that $\bar{w}_{n_{k}} \rightarrow \bar{u}$. 
Also, since $\left\{v_{n_{k}}\right\},\left\{u_{n_{k}}\right\},\left\{y_{n_{k}}\right\}$ and $\left\{\bar{w}_{n_{k}}\right\}$ are bounded, we can choose a subsequence $\left\{k_{j}\right\}$ of $\{k\}$ such that

$$
\begin{aligned}
& \frac{1}{\rho}\left[\limsup _{k \rightarrow \infty}\left(\left\|s_{n_{k}}-v_{n_{k}}\right\|^{2}-\left\|s_{n_{k}}-y_{n_{k}}\right\|^{2}\right)\right] \\
\leq & \limsup _{k \rightarrow \infty}\left[\left(\sigma-\frac{1}{\rho_{n_{k}}}\right)\left\|v_{n_{k}}-y_{n_{k}}\right\|^{2}-2\left\langle\bar{w}_{n_{k}}-u_{n_{k}}, v_{n_{k}}-y_{n_{k}}\right\rangle\right] \\
= & \lim _{j \rightarrow \infty}\left[\left(\sigma-\frac{1}{\rho_{n_{k_{j}}}}\right)\left\|v_{n_{k_{j}}}-y_{n_{k_{j}}}\right\|^{2}-2\left\langle\bar{w}_{n_{k_{j}}}-u_{n_{k_{j}}}, v_{n_{k_{j}}}-y_{n_{k_{j}}}\right\rangle\right] .
\end{aligned}
$$

Thus, we obtain from Eq. 54 that

$$
\limsup _{k \rightarrow \infty}\left(\left\|s_{n_{k}}-v_{n_{k}}\right\|^{2}-\left\|s_{n_{k}}-y_{n_{k}}\right\|^{2}\right) \leq \rho\left(\sigma-\frac{1}{\rho}\right) t .
$$

At this point, we claim that $t=0$. Otherwise, Eq. 58 will become

$$
\limsup _{k \rightarrow \infty}\left(\left\|s_{n_{k}}-v_{n_{k}}\right\|^{2}-\left\|s_{n_{k}}-y_{n_{k}}\right\|^{2}\right) \leq \rho\left(\sigma-\frac{1}{\rho}\right) t<0 .
$$

But for $\varepsilon=\frac{-\rho\left(\sigma-\frac{1}{\rho}\right)}{2} t>0$, there exists $N \in \mathbb{N}$ such that

$\left\|s_{n_{k}}-v_{n_{k}}\right\|^{2}-\left\|s_{n_{k}}-y_{n_{k}}\right\|^{2} \leq \rho\left(\sigma-\frac{1}{\rho}\right)+\varepsilon=\frac{\rho\left(\sigma-\frac{1}{\rho}\right)}{2}<0 \quad \forall k \in \mathbb{N}, \quad k \geq N$.

Thus, we obtain that

$$
\left\|v_{n_{k}}-s_{n_{k}}\right\|<\left\|y_{n_{k}}-s_{n_{k}}\right\| \quad \forall k \in \mathbb{N},
$$

which is a contradiction to the definition of $y_{n_{k}}=P_{C}\left(v_{n_{k}}-\rho_{n_{k}} u_{n_{k}}\right)$. Therefore, $t=0$. Hence, Eq. 54 becomes

$$
\lim _{k \rightarrow \infty}\left\|v_{n_{k}}-y_{n_{k}}\right\|=0 .
$$

Case 2: Suppose that $\liminf _{n \rightarrow \infty} \alpha_{n}>0$. Then, we obtain from Eq. 53 that

$$
\begin{aligned}
0 \leq \limsup _{k \rightarrow \infty}\left\|v_{n_{k}}-y_{n_{k}}\right\|^{2} & \leq \limsup _{k \rightarrow \infty}\left(\alpha_{n_{k}}\left\|v_{n_{k}}-y_{n_{k}}\right\|^{2}\right)\left(\limsup _{k \rightarrow \infty} \frac{1}{\alpha_{n_{k}}}\right) \\
& =\left(\limsup _{k \rightarrow \infty} \alpha_{n_{k}}\left\|v_{n_{k}}-y_{n_{k}}\right\|^{2}\right)\left(\frac{1}{\liminf _{k \rightarrow \infty} \alpha_{n_{k}}}\right) \\
=0 . & =0 .
\end{aligned}
$$

Therefore, we obtain that

$$
\lim _{k \rightarrow \infty}\left\|v_{n_{k}}-y_{n_{k}}\right\|=0 .
$$


Theorem 5.3 Let $\left\{x_{n}\right\}$ be a sequence generated by Algorithm 3.3. Then, under assumption (8) and Assumption 3.1, we have that $\left\{x_{n}\right\}$ converges to an element of $\Gamma$.

Proof By Lemma 4.3, $\left\{x_{n}\right\}$ is bounded. Thus, there exists a subsequence $\left\{x_{n_{k}}\right\}$ of $\left\{x_{n}\right\}$ such that $\left\{x_{n_{k}}\right\}$ converges to some point $x^{*}$. Thus, we obtain from Lemma 5.2 (b) that

$$
\lim _{k \rightarrow \infty}\left\|v_{n_{k}}-y_{n_{k}}\right\|=0
$$

Also, from Lemma 4.2, we obtain that

$$
\lim _{k \rightarrow \infty}\left\|v_{n_{k}}-x_{n_{k}}\right\|=0
$$

Hence, from Eqs. 59 and 60, we obtain

$$
\lim _{k \rightarrow \infty} y_{n_{k}}=\lim _{k \rightarrow \infty} P_{C}\left(v_{n_{k}}-\rho_{n_{k}} u_{n_{k}}\right)=\lim _{k \rightarrow \infty} x_{n_{k}}=x^{*} .
$$

Now, without loss of generality, we may assume that $\rho_{n_{k}} \rightarrow \rho^{*}$ and $u_{n_{k}} \rightarrow u^{*}$. Since $F$ is continuous, it is outer-semicontinuous. Thus, we obtain that $u^{*} \in F\left(x^{*}\right)$. Therefore, we obtain from Eq. 61 that

$$
P_{C}\left(x^{*}-\rho^{*} u^{*}\right)=x^{*} .
$$

It then follows from Lemma 2.10 that $x^{*} \in \Gamma$.

We now show that $\left\{x_{n}\right\}$ converges to $x^{*}$.

Replacing $z$ by $x^{*}$ in Lemma 4.3, we obtain that $\lim _{n \rightarrow \infty}\left\|x_{n}-x^{*}\right\|^{2}$ exists. Since $x^{*}$ is an accumulation point of $\left\{x_{n}\right\}$, we obtain that $\left\{x_{n}\right\}$ converges to $x^{*}$.

Remark 5.4 Following Remark 4.13, we can also obtain various corollaries of Theorem 5.3.

\section{Numerical Experiments}

In this section, we discuss the numerical behavior of Algorithm 3.2 and Algorithm 3.3 using test examples taken from the literature. We only compare our methods with Algorithms 1.3 and 1.4 of He et al. (2019) since in He et al. (2019, Section 4), we have that the methods in He et al. (2019) are more efficient than most relevant methods in the literature.

The codes are implemented in Matlab 2016 (b). We perform all computations on a personal computer with an Intel(R) Core(TM) i5-2600 CPU at $2.30 \mathrm{GHz}$ and 8.00 Gb-RAM.

We consider the same set of examples considered in He et al. (2019, Section 4). We randomly choose $x_{0}, x_{1} \in \mathbb{R}^{N}$ and the inertial factor $\theta_{n}$ satisfying assumptions (8) and (14). 
Example 6.1 Consider the following convex non-smooth optimization problem (see also Dong et al. 2017; He et al. 2019)

$$
\min _{x \in C} \varphi(x),
$$

where $\varphi(x)=-x_{1}+20 \max \left\{x_{1}^{2}+x_{2}^{2}-1,0\right\}$ and $C=\left\{x \in \mathbb{R}_{+}^{2}: x_{1}+x_{2} \leq 1\right\}$. This problem is equivalent to the MVIP (1) with $F(x)=\partial \varphi(x)$, where $\partial \varphi(x)$ is the subdifferential of $\varphi$ at $x$ :

$$
\partial \varphi(x)= \begin{cases}\left(-1+40 x_{1}, 40 x_{2}\right), & \text { if }\|x\|>1 \\ (-1,0), & \text { if }\|x\|<1 \\ \left\{\left(-1+40 t x_{1}, 40 t x_{2}\right) \mid t \in[0,1]\right\}, & \text { if }\|x\|=1\end{cases}
$$

Then, we see that $x^{*}=(1,0)$ is the unique solution of the problem, and the multivalued mapping $F=\partial \varphi$ satisfies the assumptions of Assumption 3.1 (b).

For the parameters, we choose $\rho_{n} \in(0,2), \sigma=0.8$, and $\gamma=0.7$. Furthermore, we take $\left\|x_{n}-x^{*}\right\| \leq \epsilon$ as the termination criterion. We stress that these choices are the same as the ones considered by He et al. (2019) for their numerical experiments.

For $\epsilon=10^{-7}$, we obtain the numerical results listed in Table 1 and Fig. 1, which show that our methods perfom better than Algorithm 1.3 and Algorithm 1.4 of $\mathrm{He}$ et al. (2019).

For $\epsilon=10^{-10}$, it was observed in He et al. (2019, Section 4) that Algorithm 1.3 of He et al. (2019) does not work well because of the presence of Procedure A in the iterative steps. Therefore, in this setting, we shall compare our methods with only Algorithm 1.4 of of He et al. (2019). For this, we obtain the numerical results reported in Table 2 and Fig. 2, which show that our methods still perform better than Algorithm 1.4 of He et al. (2019).

We consider the following cases for the numerical experiments of Example 6.1.

Case 1: $\quad x_{1}=(0.5,-0.25)^{T}, x_{0}=(0.5,-0.25)^{T}$ and $\theta_{n}=\frac{2 n-1}{8 n}$.

Case 2: $\quad x_{1}=(0.7,0.25)^{T}, x_{0}=(0.5,0.25)^{T}$ and $\theta_{n}=\frac{2 n-1}{8 n}$.

Case 3: $\quad x_{1}=(-1.5,1)^{T}, x_{0}=(1,-0.2)^{T}$ and $\theta_{n}=\frac{n-1}{n+4}$.

Case 4: $x_{1}=(-0.5,1.5)^{T}, x_{0}=(-0.5,1)^{T}$ and $\theta_{n}=\frac{n-1}{n+4}$.

Table 1 Numerical results for Example 6.1 with $\epsilon=10^{-7}$

\begin{tabular}{lccccc}
\hline & & Alg. 3.2 & Alg. 3.3 & Alg. 1.3 & Alg. 1.4 \\
\hline Case 1 & CPU time (sec) No. of Iteration & 0.07205 & 0.01503 & 0.131010 & 0.255017 \\
Case 2 & CPU time (sec) No. of Iteration & 0.07245 & 0.01513 & 0.133010 & 0.255017 \\
Case 3 & CPU time (sec) No. of Iteration & 0.07205 & 0.03504 & 0.132010 & 0.255018 \\
Case 4 & CPU time (sec) No. of Iteration & 0.04804 & 0.04304 & 0.10408 & 0.261018 \\
\hline
\end{tabular}



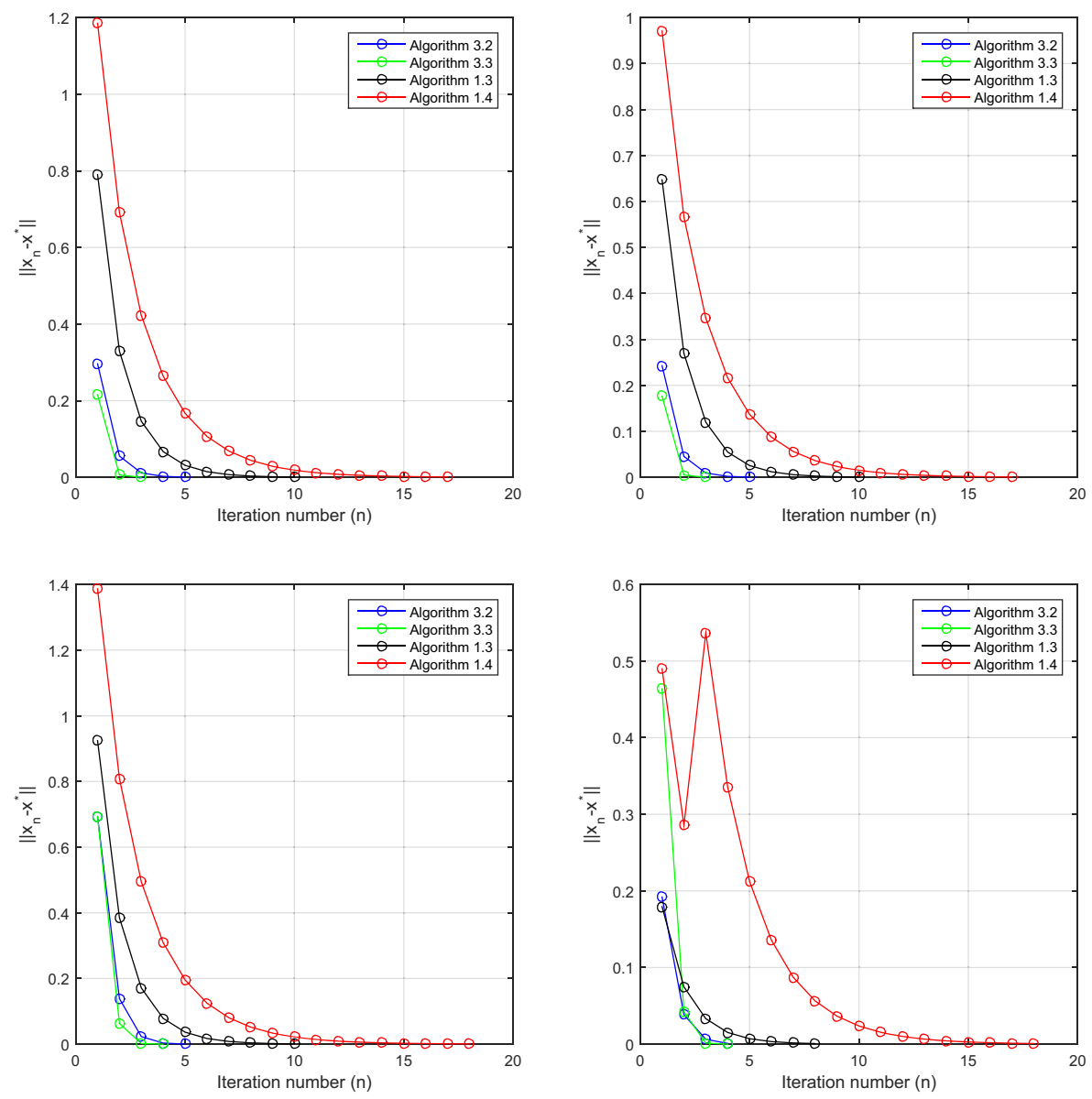

Fig. $1\left\|x_{n}-x^{*}\right\|$ vs Iteration numbers (n) for Example 6.1 with $\epsilon=10^{-7}$ : Top Left: Case 1; Top Right: Case 2; Bottom Left: Case 3; Bottom Right: Case 4

Table 2 Numerical results for Example 6.1 with $\epsilon=10^{-10}$

Alg. 3.2

Alg. 3.3

Alg. 1.4

Case 1 CPU time (sec) No. of Iteration

0.142010

0.01604

0.791034

Case 2

CPU time (sec) No. of Iteration

0.139010

0.01104

0.784034

Case 3

CPU time (sec) No. of Iteration

0.10108

0.03705

0.781034

Case 4

CPU time (sec) No. of Iteration

0.10208

0.03705

0.792035 

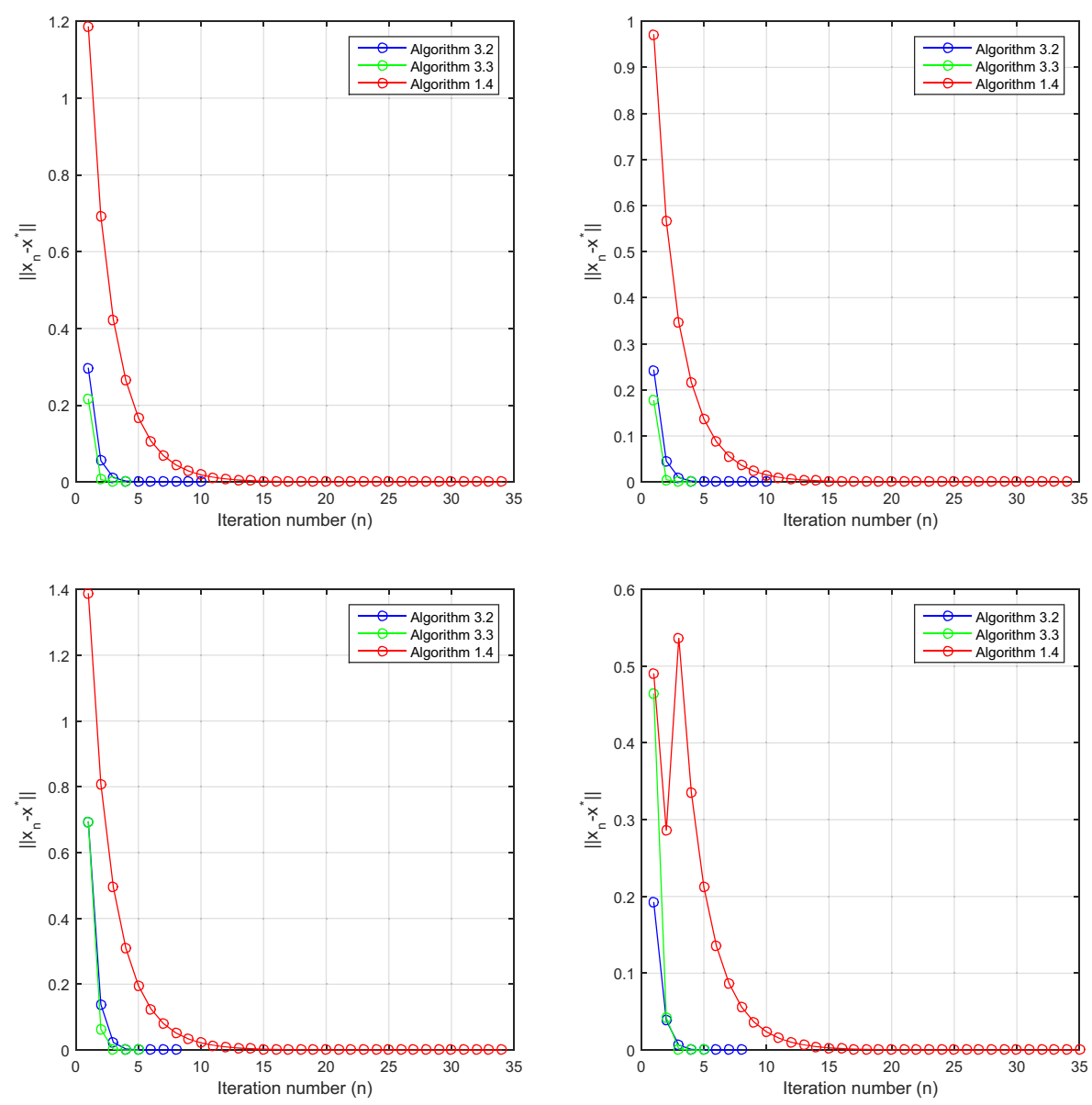

Fig. $2\left\|x_{n}-x^{*}\right\|$ vs Iteration numbers (n) for Example 6.1 with $\epsilon=10^{-10}$ : Top Left: Case 1; Top Right: Case 2; Bottom Left: Case 3; Bottom Right: Case 4

Example 6.2 We next consider the following optimization problem which was also considered in He et al. (2019) and Ye and He (2015).

$$
\min _{x \in C} \varphi(x)
$$

where $C=\left\{x \in \mathbb{R}^{5}: x_{i} \geq 0, \quad i=1,2, \cdots, 5, \sum_{i=1}^{5} x_{i}=a, a>0\right\}$ and $\varphi(x)=$ $\frac{0.5\langle H x, x\rangle+\langle q, x\rangle+1}{\sum_{i=1}^{5} x_{i}}$.

Furthermore, $H$ denotes a positive diagonal matrix with the same element $h$ taken from the interval $(0.1,2)$ and $q=(-1,-1,-1,-1,-1)$. Clearly, this problem 
Table 3 Numerical results for Example 6.2 with $\epsilon=10^{-4}$

\begin{tabular}{lllll}
\hline & & Alg. 3.2 & Alg. 3.3 & Alg. 1.4 \\
\hline Case 1 & CPU time (sec) No. of Iteration & 0.521014 & 0.06106 & 1.121042 \\
Case 2 & CPU time (sec) No. of Iteration & 0.310010 & 0.08304 & 0.922033 \\
Case 3 & CPU time (sec) No. of Iteration & 0.22009 & 0.08004 & 0.921032 \\
Case 4 & CPU time (sec) No. of Iteration & 0.29209 & 0.08704 & 0.929032 \\
\hline
\end{tabular}
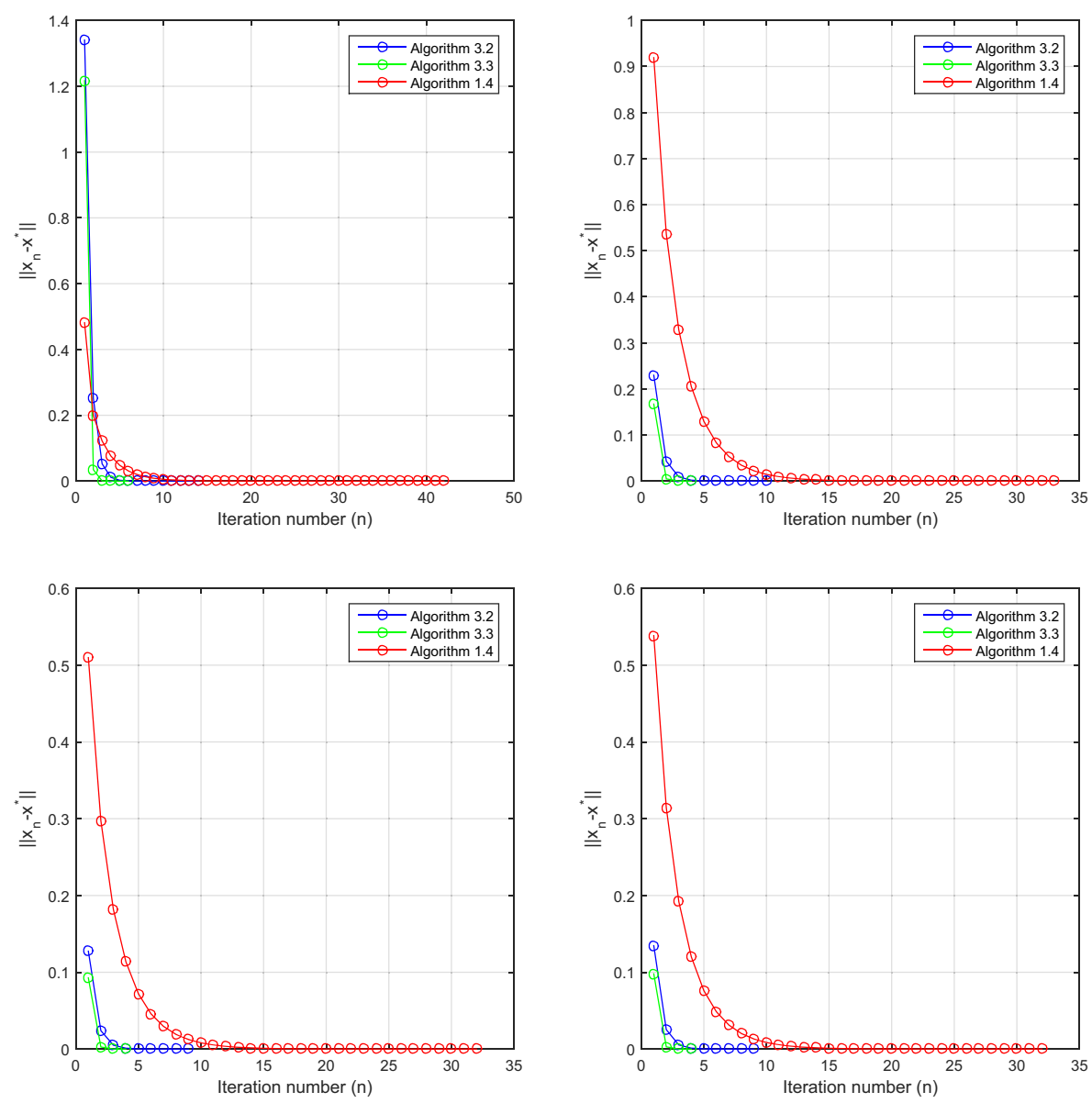

Fig. $3\left\|x_{n}-x^{*}\right\|$ vs Iteration numbers (n) for Example 6.2 with $\epsilon=10^{-4}$ : Top Left: Case 1; Top Right: Case 2; Bottom Left: Case 3; Bottom Right: Case 4 
is equivalent to MVIP (1) with solution set $\Gamma=\left\{\frac{1}{5}(a, \cdots, a)\right\}$, where $F(x)=$ $\left(\varphi_{1}(x), \cdots, \varphi_{5}(x)\right)$ and

$$
\varphi_{i}(x)=\frac{\partial \varphi(x)}{\partial x_{i}}=\frac{h x_{i} \sum_{i=1}^{5} x_{i}-0.5 h \sum_{i=1}^{5} x_{i}^{2}-1}{\left(\sum_{i=1}^{5} x_{i}\right)^{2}}
$$

For $\epsilon=10^{-4}, \sigma=0.3$ and for some randomly chosen values of $a$, we compare our methods with Algorithm 1.4 of He et al. (2019). We obtain the numerical results displayed in Table 3 and Fig. 3, which show that our methods perform better than Algorithm 1.4 of He et al. (2019).

We consider the following cases for the numerical experiments of Example 6.2.

Case 1: $\quad x_{1}=(1,0.5,1,1.5,1)^{T}, x_{0}=(1,0.5,1,1.5,1)^{T}, a=5$ and $\theta_{n}=\frac{2 n-1}{8 n}$.

Case 2: $\quad x_{1}=(3,2,2,1,2)^{T}, x_{0}=(4.3,2.5,2.2,0.3,0.7)^{T}, a=10$ and $\theta_{n}=$ $\frac{2 n-1}{8 n}$.

Case 3: $\quad x_{1}=(0.1,0.9,2,0.5,1.5)^{T}, x_{0}=(0.3,0.5,1.2,2.5,0.5)^{T}, a=5$ and $\theta_{n}=\frac{n-1}{n+4}$.

Case 4: $\quad x_{1}=(2.1,2.9,2,1.5,1.5)^{T}, x_{0}=(1.3,1.5,2.2,3.5,1.5)^{T}, a=10$ and $\theta_{n}=\frac{n-1}{n+4}$.

\section{Conclusion}

We propose two new inertial extrapolation projection-type methods for solving MVIPs when the multivalued mapping $F$ is only required to be locally bounded without any monotonicity assumption. The first method uses a linesearch as in He et al. (2019, Algorithms 1 and 2) while the second method uses a different linesearch procedure with the aim of minimizing the number of evaluation of the multivalued mapping $F$ in each search. Furthermore, our inertial techniques for establishing the convergence of these methods are quite different from the commonly used ones in most papers (see for example Cholamjiak et al. 2018; Chuang 2017; Ochs et al. 2015; Lorenz and Pock 2015; Polyak 1964; Shehu and Cholamjiak 2019; Lorenz and Pock 2015; Mainge 2008; Moudafi and Oliny 2003; Shehu et al. 2019; Shehu et al. 2019; Thong and Hieu 2018; Thong and Hieu 2017 and the references therein). Moreover, based on the weaker assumptions on the inertial factor in our methods, we derive several special cases of our methods. Finally, we consider some numerical implementations of our methods and compare them with the methods in He et al. (2019, Algorithms 1 and 2), in order to show the profits that we gain by introducing the new inertial extrapolation steps. In fact, in all our comparisons, the numerical results demonstrate that our methods perform better than the methods in He et al. (2019, Algorithms 1 and 2). Thus, our results improve and generalize many recent important results in this direction. 
Acknowledgements The authors sincerely thank the Editor-in-Chief and anonymous referees for their careful reading, constructive comments and fruitful suggestions that help improve the manuscript. The research of the first author is supported by the National Research Foundation (NRF) South Africa (S\& F-DSI/NRF Free Standing Postdoctoral Fellowship; Grant Number: 120784). The first author also acknowledges the financial support from DSI/NRF, South Africa Center of Excellence in Mathematical and Statistical Sciences (CoE-MaSS) Postdoctoral Fellowship. The second author has received funding from the European Research Council (ERC) under the European Union's Seventh Framework Program (FP7 - 2007-2013) (Grant agreement No. 616160).

Funding Open Access funding provided by Institute of Science and Technology (IST Austria).

Open Access This article is licensed under a Creative Commons Attribution 4.0 International License, which permits use, sharing, adaptation, distribution and reproduction in any medium or format, as long as you give appropriate credit to the original author(s) and the source, provide a link to the Creative Commons licence, and indicate if changes were made. The images or other third party material in this article are included in the article's Creative Commons licence, unless indicated otherwise in a credit line to the material. If material is not included in the article's Creative Commons licence and your intended use is not permitted by statutory regulation or exceeds the permitted use, you will need to obtain permission directly from the copyright holder. To view a copy of this licence, visit http://creativecommonshorg/licenses/by/4. $0 \%$

\section{References}

Alvarez F, Attouch H (2001) An inertial proximal method for maximal monotone operators via discretization of a nonlinear oscillator with damping. Set-Valued Anal 9:3-11

Attouch H, Cabot A (2019) Convergence of a relaxed inertial proximal algorithm for maximally monotone operators. Math Program. https://doi.org/10.1007/s 10107-019-01412-0

Attouch H, Cabot A (2019) Convergence of a relaxed inertial forward-backward algorithm for structured monotone inclusions. Appl Math Optim 80:547-598

Attouch H, Goudon X, Redont P (2000) The heavy ball with friction. I. The continuous dynamical system. Commun Contemp Math 2(1):1-34

Beck A, Teboulle M (2009) A fast iterative shrinkage-thresholding algorithm for linear inverse problems. SIAM J Imaging Sci 2(1):183-202

Bot RI, Csetnek ER, Hendrich C (2015) Inertial Douglas-Rachford splitting for monotone inclusion. Appl Math Comput 256:472-487

Bot RI, Csetnek ER (2016) An inertial alternating direction method of multipliers. Minimax Theory Appl $1: 29-49$

Brouwer LEJ (1912) Über Abbildung von Mannigfaltigkeiten. Math Ann 71(4):97-115

Browder FE (1965) Multi-valued monotone nonlinear mappings and duality mappings in Banach spaces. Trans Am Math Soc 18:338-351

Carey M, Ge YE (2012) Comparison of methods for path flow reassignment for dynamic user equilibrium. Netw Spat Econ 12:337-376

Censor Y, Gibali A, Reich S (2011) The subgradient extragradient method for solving variational inequalities in Hilbert space. J Optim Theory Appl 148:318-335

Chambolle A, Dossal C. h. (2015) On the convergence of the iterates of the "fast iterative shrinkage/thresholding algorithm". J Optim Theory Appl 166:968-982

Cholamjiak W, Cholamjiak P, Suantai S (2018) An inertial forward-backward splitting method for solving inclusion problems in Hilbert spaces. J Fixed Point Theory Appl, 20. https://doi.org/10.1007/ s11784-018-0526-5

Chuang CS (2017) Hybrid inertial proximal algorithm for the split variational inclusion problem in Hilbert spaces with applications. Optimization 66(5):777-792

Dong QL, Lu YY, Yang J, He S (2017) Approximately solving multi-valued variational inequalities by using a projection and contraction algorithm. Numer Algor 76:799-812 
Facchinei F, Pang JS (2003) Finite-dimensional variational inequalities and complementarity problems vol $1 \& 2$. Springer, Berlin

Fang CJ, Chen SL (2014) A subgradient extragradient algorithm for solving multi-valued variational inequality. Appl Math Comput 229:123-130

Goebel K, Reich S (1984) Uniform convexity, hyperbolic geometry, and nonexpansive mappings. Marcel Dekker, New York

He YR (2006) A new double projection algorithm for variational inequalities. J Comput Appl Math 185:66-173

He X, Huang N, Li X (2019) Modified projection methods for solving multi-valued variational inequality without monotonicity. Netw Spat Econ. https://doi.org/10.1007/s11067-019-09485-2

Konnov IV (1998) A combined relaxation method for variational inequalities with nonlinear constraints. Math Program 80:239-252

Korpelevich GM (1976) The extragradient method for finding saddle points and other problems. Matecon 12:747-756

Lorenz DA, Pock T (2015) An inertial forward-backward algorithm for monotone inclusions. J Math Imaging Vis 51:311-325

Mainge PE (2008) Convergence theorems for inertial KM-type algorithms. J Comput Appl Math 219(1):223-236

Moudafi A, Oliny M (2003) Convergence of a splitting inertial proximal method for monotone operators. J Comput Appl Math 155:447-454

Nadler SB (1969) Multi-valued contraction mappings. Pac J Math (English Series) 30:475-488

Nesterov Y (1983) A method of solving a convex programming problem with convergence rate $\mathrm{O}\left(1 / k^{2}\right)$. Soviet Math Doklady 27:372-376

Ochs P, Brox T, Pock T (2015) iPiasco: inertial proximal algorithm for strongly convex optimization. J Math Imaging Vis 53:171-181

Oggioni G, Smeers Y, Allevi E, Schaible S (2012) A generalized Nash equilibrium model of market coupling in the European power system. Netw Spat Econ 12:503-560

Polyak BT (1964) Some methods of speeding up the convergence of iterates methods. USSR Comput Math Phys 4(5):1-17

Raciti F, Falsaperla P (2007) Improved noniterative algorithm for the calculation of the equilibrium in the traffic network problem. J Optim Theory Appl 133:401-411

Rockafellar RT, Wets JB (2004) Variational analysis. Springer, New York

Rockafellar RT (1970) Convex analysis. Princeton University Press, Princeton

Shehu Y, Cholamjiak P (2019) Iterative method with inertial for variational inequalities in Hilbert spaces. Calcolo, 56(1)

Shehu Y, Li XH, Dong QL (2019) An efficient projection-type method for monotone variational inequalities in Hilbert spaces. Numer Algorithms. https://doi.org/10.1007/s11075-019-00758-y

Shehu Y, Vuong PT, Zemkoho A (2019) An inertial extrapolation method for convex simple bilevel optimization. Optim Methods Softw. https://doi.org/10.1080/10556788.2019.1619729

Thong DV, Hieu DV (2018) Inertial subgradient extragradient algorithms with line-search process for solving variational inequality problems and fixed point problems. Numer Algorithms. https://doi.org/10.1007/s11075-018-0527-x

Thong DV, Hieu DV (2017) An inertial method for solving split common fixed point problems. J Fixed Point Theory Appl 19(4):3029-3051

Tseng P (2000) A modified forward-backward splitting method for maximal monotone mappings. SIAM J Control Optim 38:431-446

Vuong PT (2019) The global exponential stability of a dynamical system for solving variational inequalities. Netw Spat Econ. https://doi.org/10.1007/s11067-019-09457-6

Ye ML, He YR (2015) A double projection method for solving variational inequalities without monotonicity. Comput Optim Appl 60:141-150

Publisher's Note Springer Nature remains neutral with regard to jurisdictional claims in published maps and institutional affiliations. 